Supporting information

\title{
Self-assembled copper metallogel bearing terpyridine and the application as catalyst for click reaction in water
}

\author{
Tong Zhao, Shaorui Chen*, Kai Kang, Jvjie Ren and Xudong Yu*
}

College of Science, Hebei University of Science and Technology, Shijiazhuang 050018, Hebei,

\section{China}

E-mail for corresponding author: sjz_wgq@126.com (S. Chen); chemyxd@163.com (X.Yu)

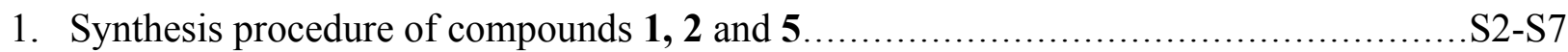

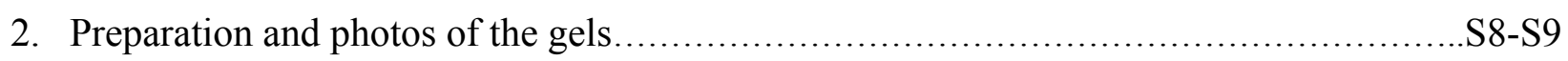

3. g parameter, UV-vis, EPR spectra and fluorescent spectra of the solutions and the gels..S10$\mathrm{S} 12$

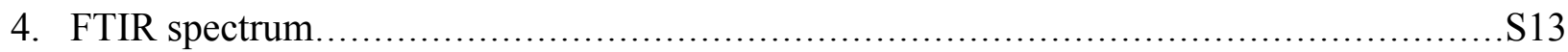

5. SEM, XRD, rheological and other data of the xerogels.............................S14-S16

6. IR, ${ }^{1} \mathrm{H}$ NMR and ${ }^{13} \mathrm{C}$ NMR spectra of the 1,2,3-triazole derivatives ..................S17-S29 


\section{Synthesis procedure}

Compounds 1, 2 and 5 were prepared according to the literature, ${ }^{1-3}$ respectively.
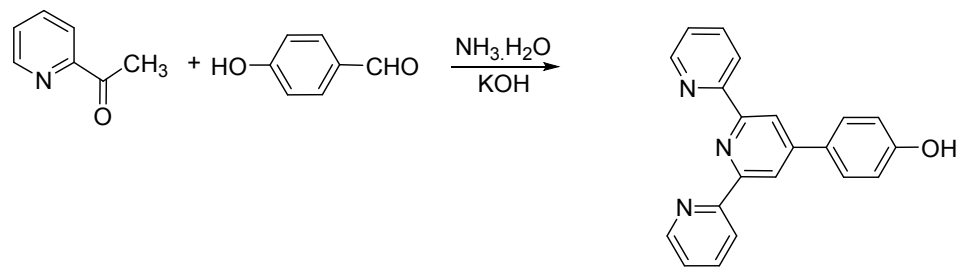

\section{Synthesis of 4-(4-Hydroxyphenyl)-2,2́,6́,2"-terpyridine (1)}

4-Hydroxybenzaldehyde (3.7 g, $30 \mathrm{mmol})$ was dissolved in $30 \mathrm{~mL}$ of ethanol solution, following addition of 2-acetylpyridine (7.3 g, $60 \mathrm{mmol})$ and $\mathrm{NH}_{3} \cdot \mathrm{H}_{2} \mathrm{O}(40 \mathrm{ml}, 25 \%)$. After stirring for a few minutes, $33 \mathrm{wt} \% \mathrm{KOH}$ solution $(90 \mathrm{mmol}, 11.7 \mathrm{~mL})$ was added dropwise to the solution and the mixture was stirred at $50{ }^{\circ} \mathrm{C}$ covernight. A large amount of green solid was obtained when the $\mathrm{pH}$ of the solution was changed to 3-4. The precipitate was filtrated and washed with methanol $(10 \mathrm{ml})$ and recrystallized with methanol for several times. A pale yellow solid was obtained after drying and the yield was $44.6 \%(4.4 \mathrm{~g})$, m.p. $>250^{\circ} \mathrm{C}$; IR(KBr): $3429,3053,1609$, 1578, 1433, 1232, $799 \mathrm{~cm}^{-1} ; \quad{ }^{1} \mathrm{H}$ NMR (500 MHz, $\left.\mathrm{CDCl}_{3}\right)$ 8: 9.08 (m, 4H, 6,6”-H, 3,3”-H, tpy), 8.91(s, 2H, 3',5'-H, tpy), 8.72 (td, 2H, $J=7.8,1.2 \mathrm{~Hz}, 4,4$ '-H, tpy), 8.14(td, 2H, $J=7.8,1.2 \mathrm{~Hz}, 5,5$ '-H, tpy), 8.02 (d, 2H, $J=9.0 \mathrm{~Hz}, 3,5-\mathrm{H}, \mathrm{ph}), 7.05(\mathrm{~d}, 2 \mathrm{H}, J=9.0 \mathrm{~Hz}, 2,6-\mathrm{H}, \mathrm{ph})$.

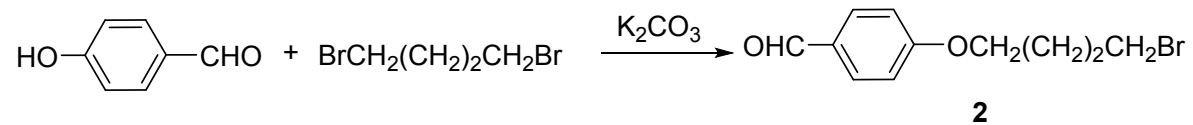

\section{Synthesis of 4-(4-bromobutoxy)-benzaldehyde (2)}

p-hydroxybenzaldehyde $(2.4 \mathrm{~g}, 20.0 \mathrm{mmol})$ and 1,4-dibromobutane $(4.8 \mathrm{~mL}, 40.0 \mathrm{mmol})$ were dissolved in dried $40 \mathrm{~mL}$ DMF, following addition of potassium carbonate $(4.1 \mathrm{~g}, 30.0 \mathrm{mmol})$, and the mixture was stired at room temperature for 24 hours. Then the reaction was quenched with water and extracted with dichloromethane. 
The organic layer was dried with anhydrous magnesium sulphate. Silica gel chromatography with petroleum ether/ ethyl acetate $(10 / 1, \mathrm{v} / \mathrm{v})$ gave compound 2 as white solid with the yield of $56.9 \%(2.9 \mathrm{~g})$. m.p. $32-34^{\circ} \mathrm{C}$; ${ }^{1} \mathrm{H}$ NMR (500 MHz, $\mathrm{CDCl}_{3}$, Fig S1) $\delta: 9.88(\mathrm{~s}, 1 \mathrm{H},-\mathrm{CHO}), 7.83$ (d, $\left.J=8.5 \mathrm{~Hz}, 2 \mathrm{H}, \mathrm{ph}\right), 6.99$ (d, $J=8.5 \mathrm{~Hz}, 2 \mathrm{H}$, ph), $4.08\left(\mathrm{t}, J=6.0 \mathrm{~Hz}, 2 \mathrm{H},-\mathrm{OCH}_{2^{-}}\right), 3.49\left(\mathrm{t}, J=6.0 \mathrm{~Hz}, 2 \mathrm{H},-\mathrm{CH}_{2} \mathrm{Br}\right), 2.10-2.07\left(\mathrm{~m}, 2 \mathrm{H},-\mathrm{CH}_{2}\right), 1.98-2.00(\mathrm{~m}$, $\left.2 \mathrm{H},-\mathrm{CH}_{2}\right)$.

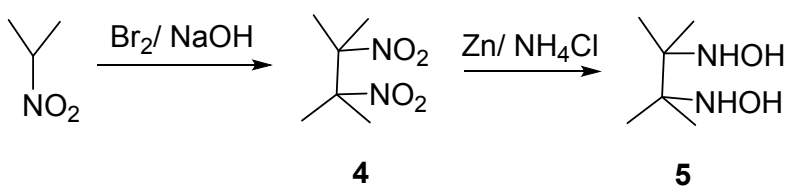

\section{Synthesis of 2, 3-dimethyl-2, 3-dinitrobutane (4)}

Under ice bath, 2-nitropropane (34.5 g, $0.39 \mathrm{~mol})$ in $65 \mathrm{~mL} 6 \mathrm{M} \mathrm{NaOH}$ solution was stirred for $30 \mathrm{~min}$, then $10 \mathrm{~mL}(0.19 \mathrm{~mol}) \mathrm{Br}_{2}$ was added drop by drop in one hour. Ethanol $(128 \mathrm{~mL})$ was added to the mixture and then refluxed for $5 \mathrm{~h}$. The mixture was poured into $300 \mathrm{~mL}$ ice water. White precipitate was formed immediately and the mixture was stirred for 10 minutes, then the white precipitate was filtered off by suction. Collected precipitate was washed with water twice then dried overnight to obtain $24.3 \mathrm{~g}$ of white powder with the yield $70.6 \%$, which was used in the next step without further purified.

\section{Synthesis of 2,3-bis(hydroxylamino)-2,3-dimethylbutane (5)}

Under $\mathrm{N}_{2}$ atmosphere, compound 2, 3-dimethyl-2, 3-dinitrobutane ( $\left.8.8 \mathrm{~g}, 0.05 \mathrm{~mol}\right)$ was dissolved in a mixture of tetrahydrofuran $(150 \mathrm{~mL})$ and water $(25 \mathrm{~mL})$. Zn powder $(13.5 \mathrm{~g})$ was added all at once to this solution cooled to $8-10{ }^{\circ} \mathrm{C}$ in an ice bath. A solution of $\mathrm{NH}_{4} \mathrm{Cl}(21.5 \mathrm{~g}, 0.4 \mathrm{~mol})$ in $\mathrm{H}_{2} \mathrm{O}(75 \mathrm{~mL})$ was added dropwise to this slurry within two hours that the temperature of the reaction did not exceed $10^{\circ} \mathrm{C}$. Then stirring was continued under $10{ }^{\circ} \mathrm{C}$ for one hour, and the flask stored in a fridge $\left(4-6{ }^{\circ} \mathrm{C}\right)$ for $16 \mathrm{~h}$. The slurry was filtered, and the 
precipitate carefully washed with THF. The solution was evaporated under vacuo until THF ceased to distill off. Sodium carbonate $(12.5 \mathrm{~g})$ and sodium chloride $(8.0 \mathrm{~g})$ were added to the viscous fluid to saturate it. Continuous extraction with chloroform $(100 \mathrm{~mL})$ was performed over 24 hours. A white powder was obtained $(2.0 \mathrm{~g}, 27.1 \%$, m.p. $180-182{ }^{\circ} \mathrm{C}$ lit. $\left.182{ }^{\circ} \mathrm{C}\right) .{ }^{1} \mathrm{H}$ NMR (500 MHz, DMSO- $d_{6}$, FigS2) $\delta(\mathrm{ppm}): 6.93$ (s, 2H, -OH), 5.38 (s, 2H, NH-), $0.99\left(\mathrm{~s}, 12 \mathrm{H},-\mathrm{CH}_{3}\right)$.

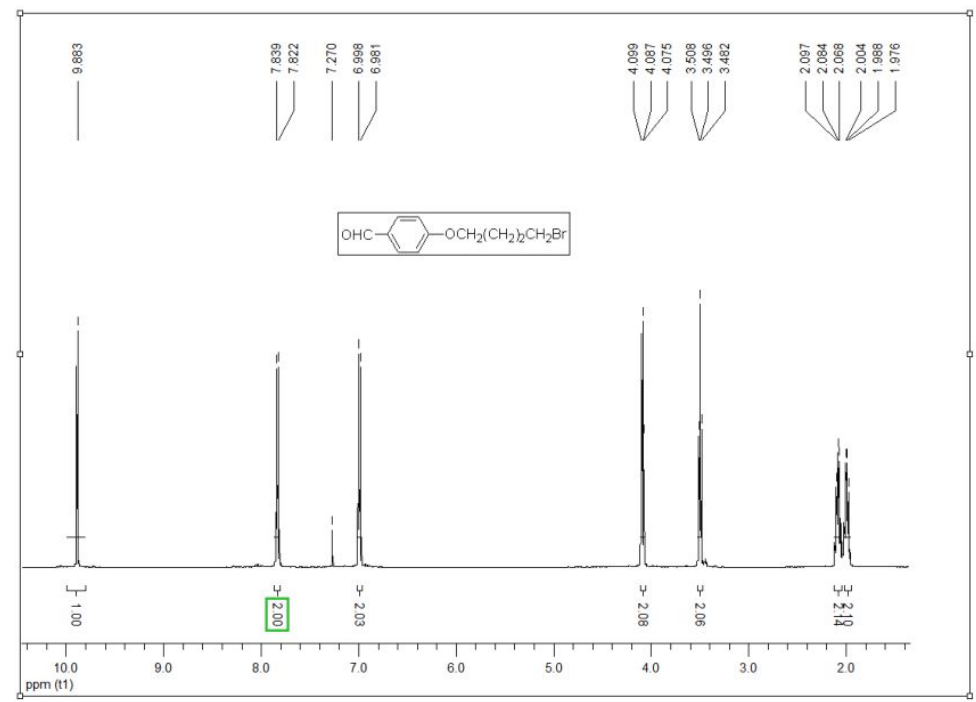

Figure S1 ${ }^{1} \mathrm{H}$ NMR spectra of compound 2

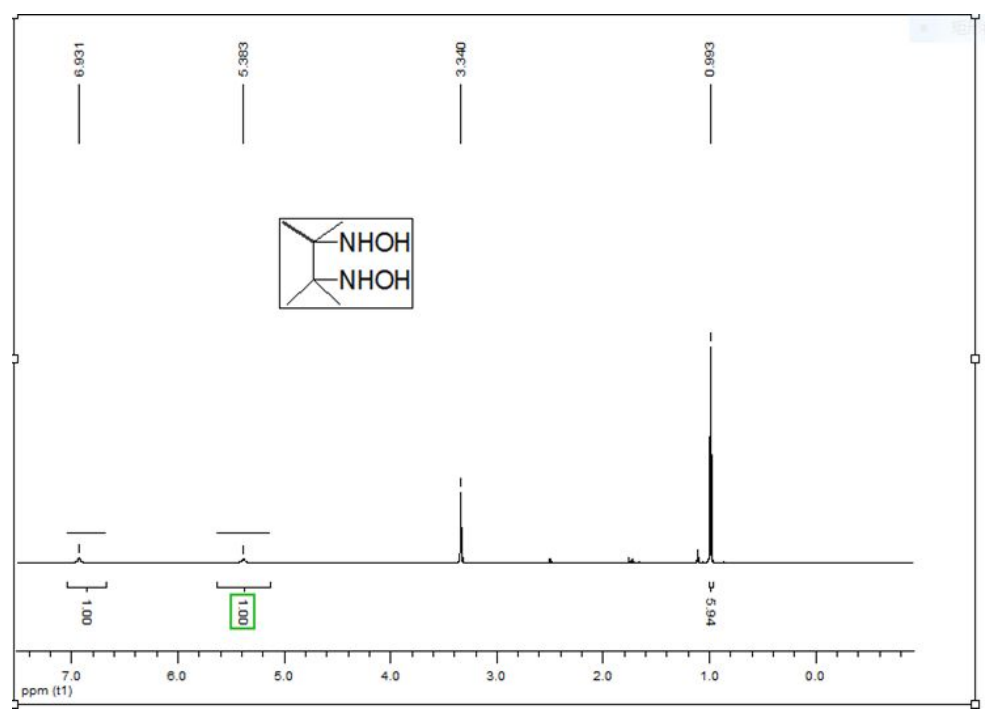


Figure $\mathbf{S 2}{ }^{1} \mathrm{H}$ NMR spectra of compound 5

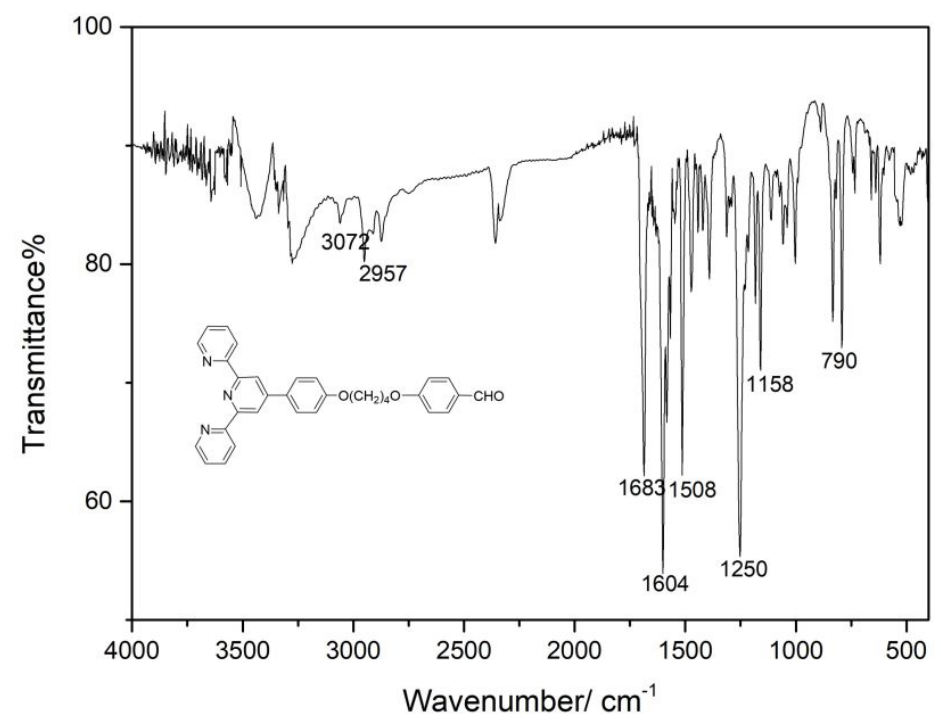

Figure S3 IR spectra of compound 3

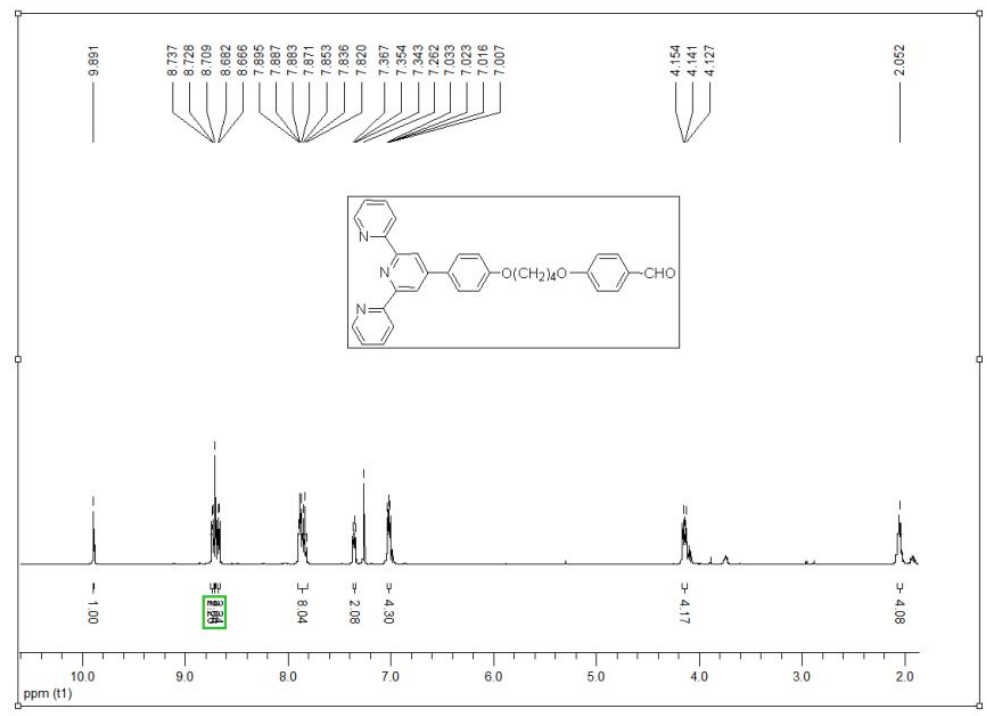

Figure S4 ${ }^{1} \mathrm{H}$ NMR spectra of compound 3 


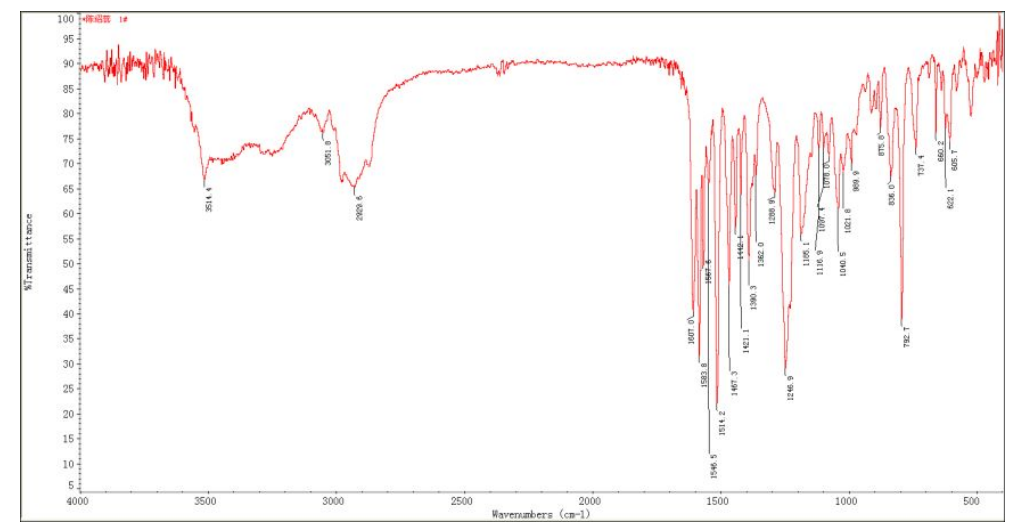

Figure S5 IR spectra of compound TPDA

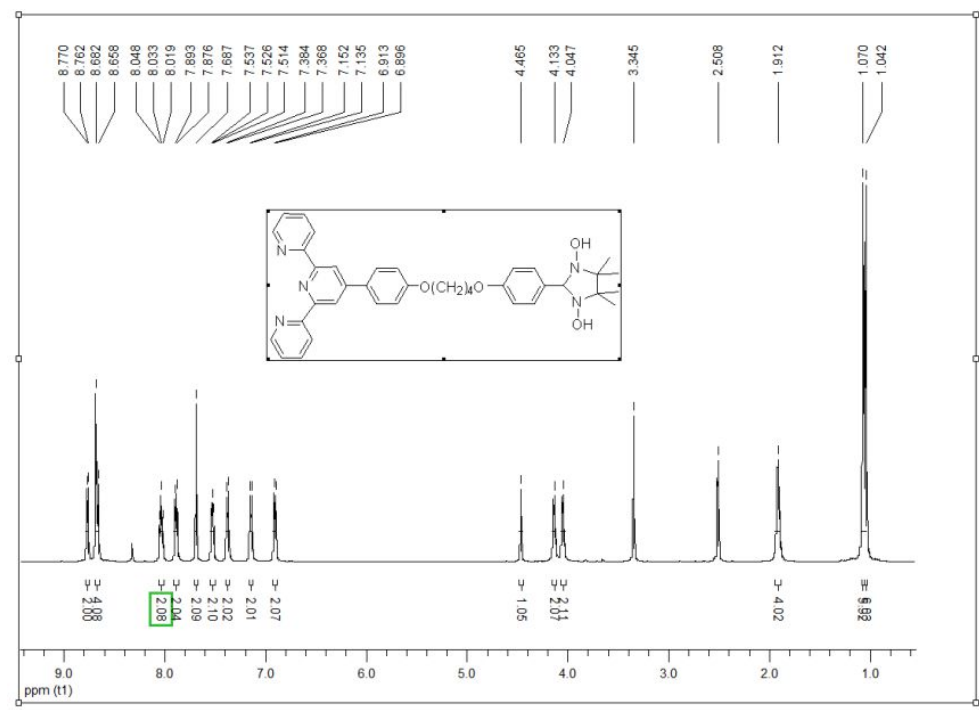

Figure S6 ${ }^{1} \mathrm{H}$ NMR spectra of compound TPDA 


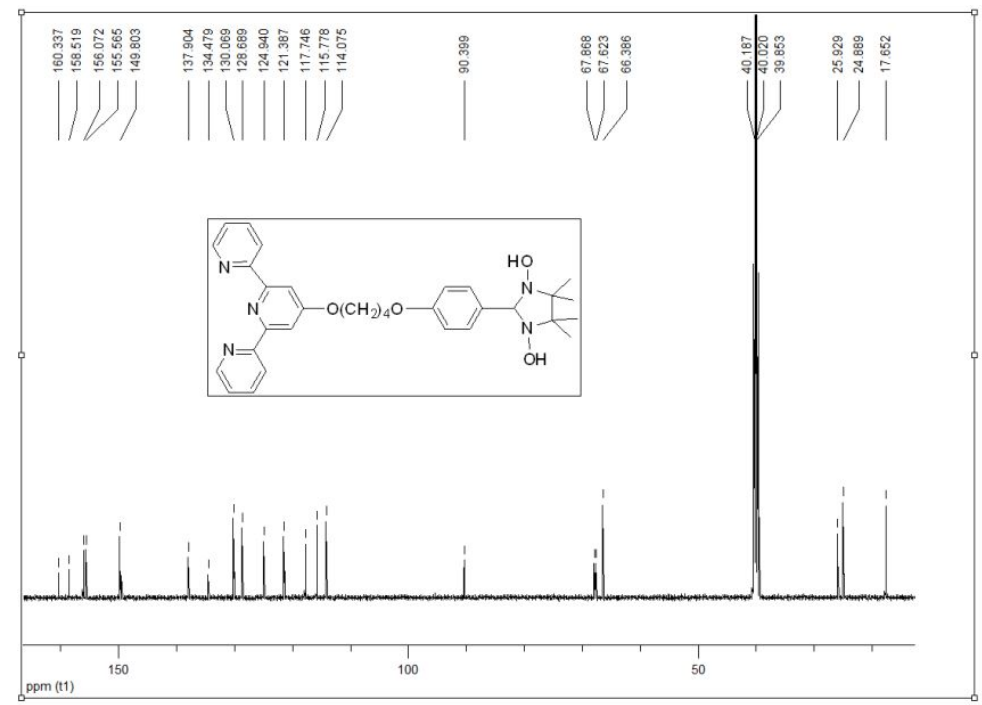

Figure $\mathbf{S} 7{ }^{13} \mathrm{C}$ NMR spectra of compound TPDA

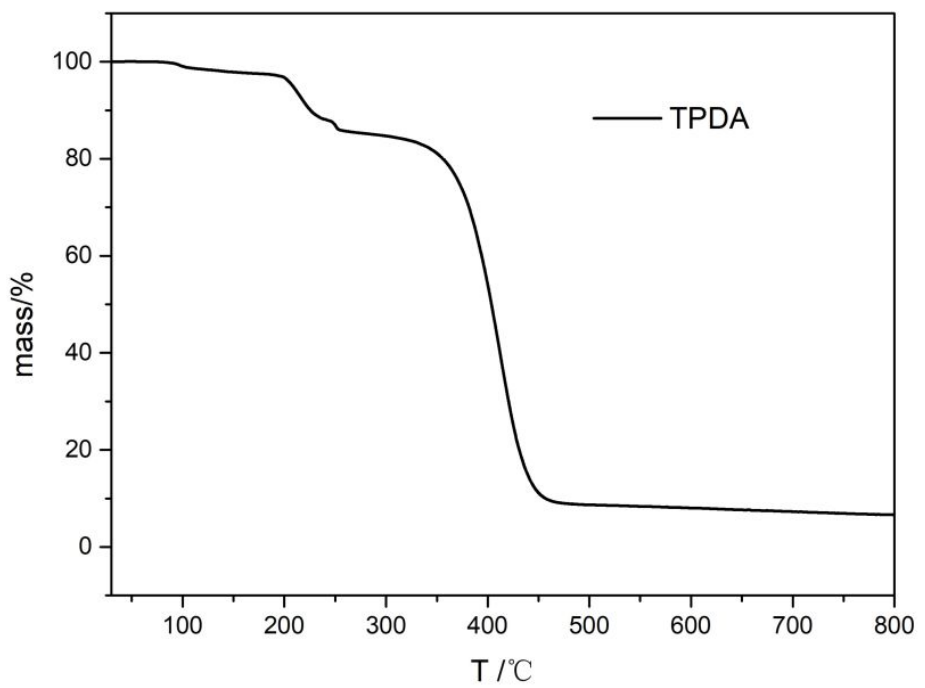


Figure S8 Thermogravimetric (TG) of compound TPDA

Table S1 The gelation test of TPDA $(25 \mathrm{mg} / \mathrm{mL})$ in different solvents

\begin{tabular}{ccc}
\hline solvent & $\mathrm{H}-\mathrm{C}$ & $\mathrm{U}$ \\
\hline methanol & $\mathrm{I}$ & $\mathrm{I}$ \\
DMSO & $\mathrm{S}$ & $\mathrm{S}$ \\
DMF & $\mathrm{S}$ & $\mathrm{S}$ \\
THF & $\mathrm{P}$ & $\mathrm{I}$ \\
chloroform & $\mathrm{P}$ & $\mathrm{I}$ \\
dichloromethane & $\mathrm{P}$ & $\mathrm{I}$ \\
$\mathrm{H}_{2} \mathrm{O}$ & $\mathrm{I}$ & $\mathrm{I}$ \\
\hline
\end{tabular}

Note: S, solution; I, insoluble; G, gel; P, precipitate; U: ultrasound; H-C: heatingcooling process.

Table S2 The gelation properties of TPDA $(25 \mathrm{mg} / \mathrm{mL})$ with different metal ion in water

\begin{tabular}{ccc}
\hline Metal salt & $\mathrm{H}-\mathrm{C}$ & $\mathrm{U}$ \\
\hline $\mathrm{Ni}\left(\mathrm{NO}_{3}\right)_{2}$ & $\mathrm{I}$ & $\mathrm{I}$ \\
$\mathrm{MgSO}_{4}$ & $\mathrm{I}$ & $\mathrm{I}$ \\
$\mathrm{LiCl}$ & $\mathrm{I}$ & $\mathrm{I}$ \\
$\mathrm{Cd}\left(\mathrm{NO}_{3}\right)_{2}$ & $\mathrm{I}$ & $\mathrm{I}$ \\
$\mathrm{BaCl}_{2}$ & $\mathrm{I}$ & $\mathrm{I}$ \\
$\mathrm{SnCl}_{2}$ & $\mathrm{I}$ & $\mathrm{I}$ \\
$\mathrm{SnCl}_{4}$ & $\mathrm{I}$ & $\mathrm{I}$ \\
$\mathrm{Pb}\left(\mathrm{NO}_{3}\right)_{2}$ & $\mathrm{I}$ & $\mathrm{I}$ \\
$\mathrm{KNO}_{3}$ & $\mathrm{I}$ & $\mathrm{I}$ \\
$\mathrm{NaNO}_{3}$ & $\mathrm{I}$ & $\mathrm{I}$ \\
$\mathrm{CoCl}_{2}$ & $\mathrm{I}$ & $\mathrm{I}$ \\
$\mathrm{Fe}\left(\mathrm{NO}_{3}\right)_{3}$ & $\mathrm{I}$ & $\mathrm{I}$ \\
$\mathrm{ZnSO}_{4}$ & $\mathrm{I}$ & $\mathrm{I}$ \\
$\mathrm{CuSO}_{4}$ & $\mathrm{G}$ & $\mathrm{G}$ \\
$\mathrm{CuCl}$ & $\mathrm{I}$ & $\mathrm{G}$ \\
$\mathrm{CuCl}_{2}$ & $\mathrm{G}$ & $\mathrm{G}$ \\
$\mathrm{CuBr}_{2}$ & $\mathrm{I}$ & $\mathrm{G}$ \\
$\mathrm{CuI}_{2}$ & $\mathrm{I}$ & $\mathrm{I}$ \\
$\left.\mathrm{Cu}_{(\mathrm{OAc}}\right)_{2}$ & $\mathrm{I}$ & $\mathrm{I}$
\end{tabular}




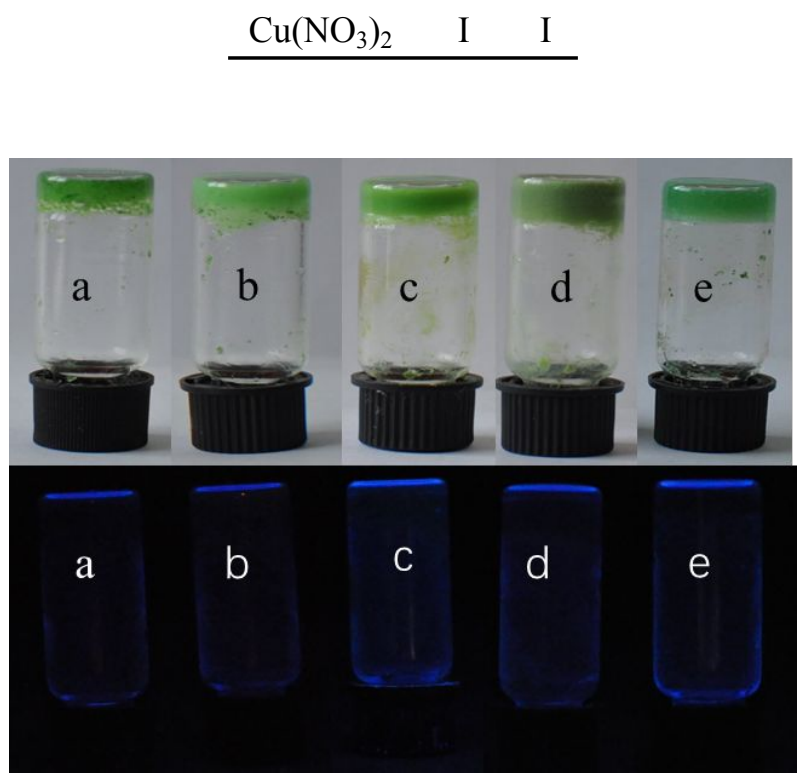

Figure S9 The gel photos of TPDA with different copper salts in water in light and in dark (irradiated by 365 nm) a: $\mathrm{CuCl}_{2}(\mathrm{H}-\mathrm{C})$, b: $\mathrm{CuCl}_{2}(\mathrm{U}), \mathrm{c}: \mathrm{CuCl}(\mathrm{U}), \mathrm{d}: \mathrm{CuBr}_{2}(\mathrm{U})$, e: $\mathrm{CuSO}_{4}(\mathrm{H}-\mathrm{C})$

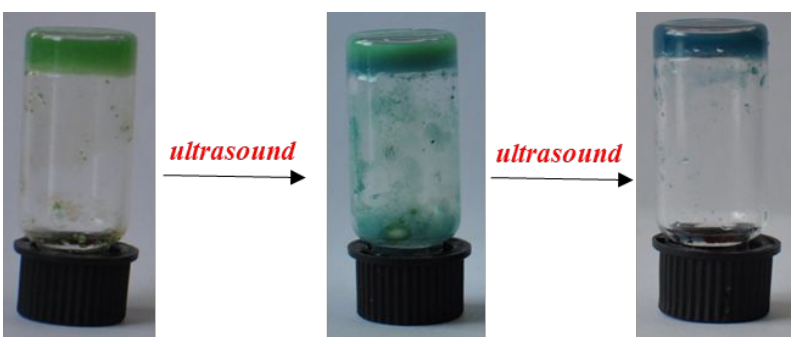

Figure S10 Photos of metallogel (TPDA/CuSO 4$)$ in water by ultrasound

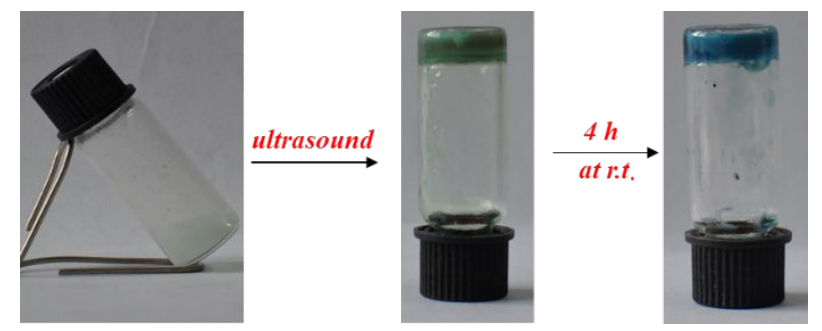

Figure S11 Photos of metallogel (TPDA $\left./ \mathrm{CuSO}_{4}\right)$ in $\mathrm{THF} / \mathrm{H}_{2} \mathrm{O}(\mathrm{v}: \mathrm{v}=3: 1)$ by ultrasound 


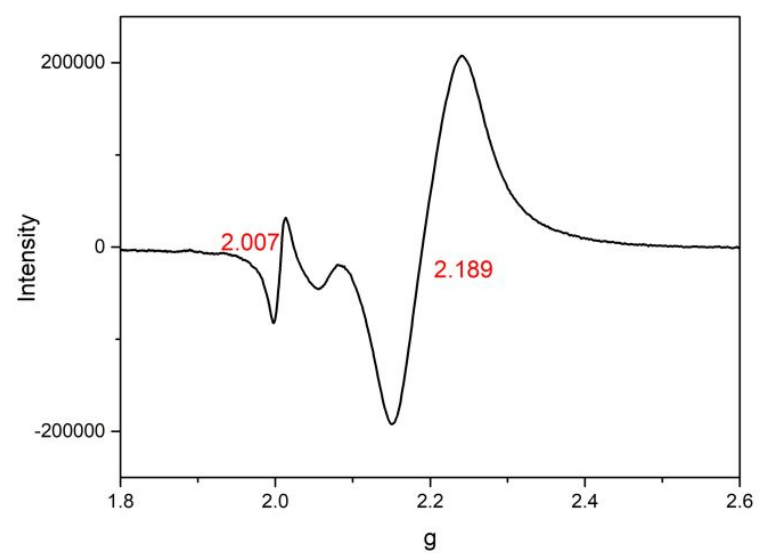

Figure S12 g parameter spectra of the gel(U)

$$
\begin{aligned}
& \mathrm{H}_{2} \mathrm{O} \stackrel{(\mathrm{II)}}{\longrightarrow} \mathrm{H} \cdot+\mathrm{HO} \cdot \\
& \mathrm{HO}+\mathrm{HO} \cdot \longrightarrow \mathrm{H}_{2} \mathrm{O}_{2} \\
& \mathrm{H} \cdot+\mathrm{O}_{2} \longrightarrow \cdot \mathrm{HO}_{2} \\
& \mathrm{H} \cdot+\cdot \mathrm{HO}_{2} \longrightarrow \mathrm{H}_{2} \mathrm{O}_{2}
\end{aligned}
$$
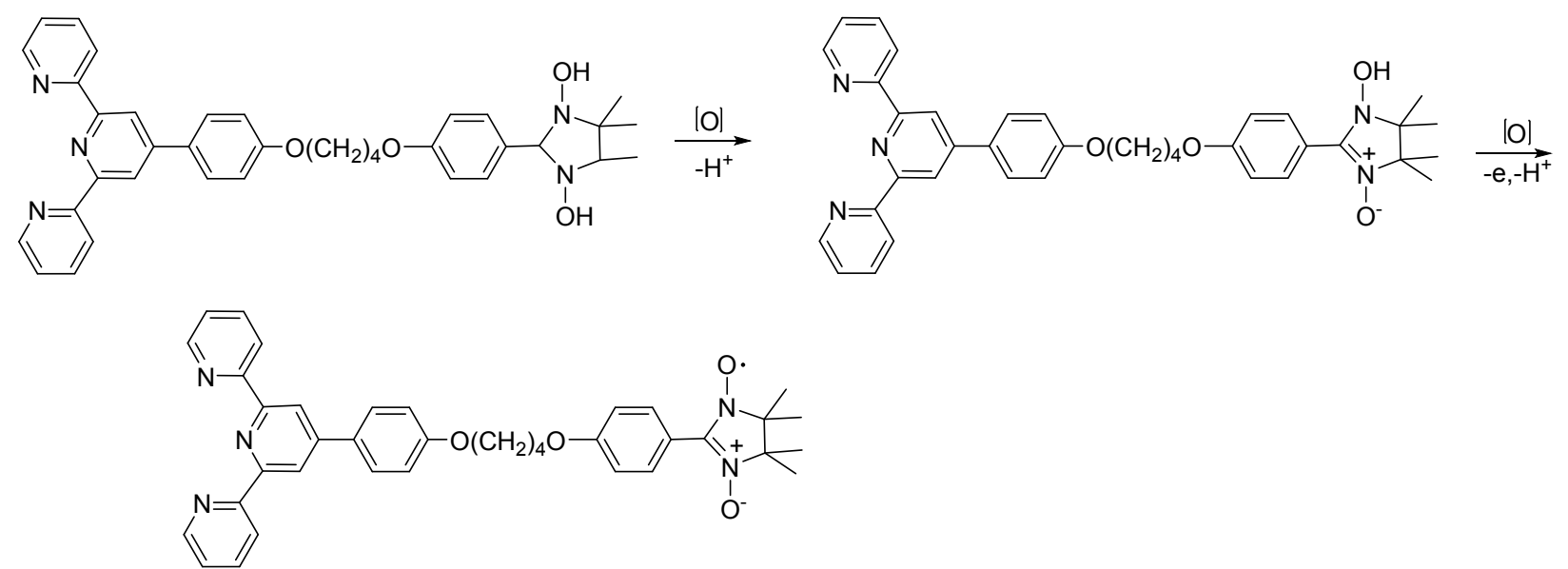

Scheme S1 Possible mechanism of the formation of the nitroxide radicals in water by ultrasound 


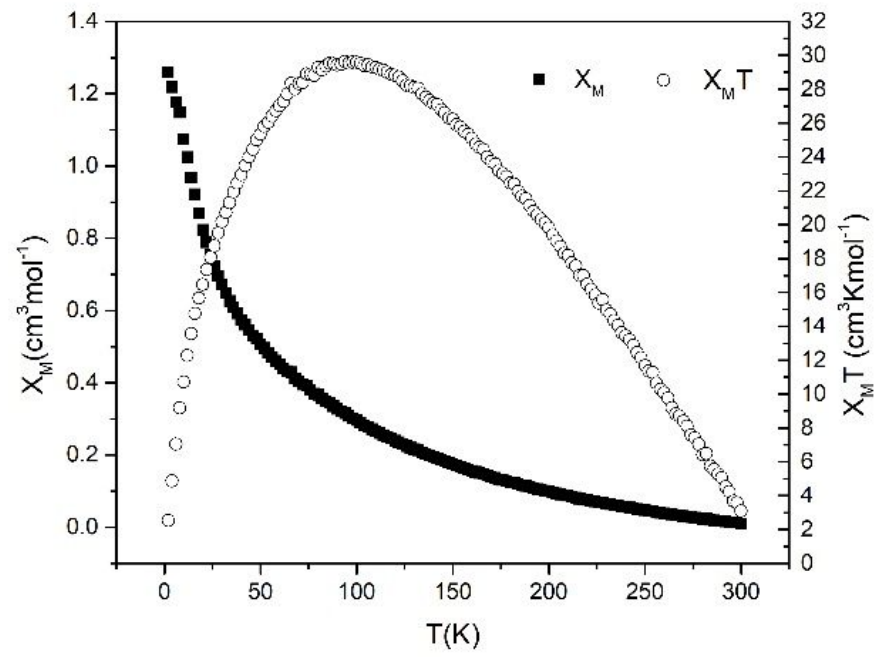

Figure S13 Temperature-dependence of $\mathrm{X}_{\mathrm{M}} \mathrm{T}$ for the xerogel-2 (the xerogel of the gel formed in water by ultrasound)
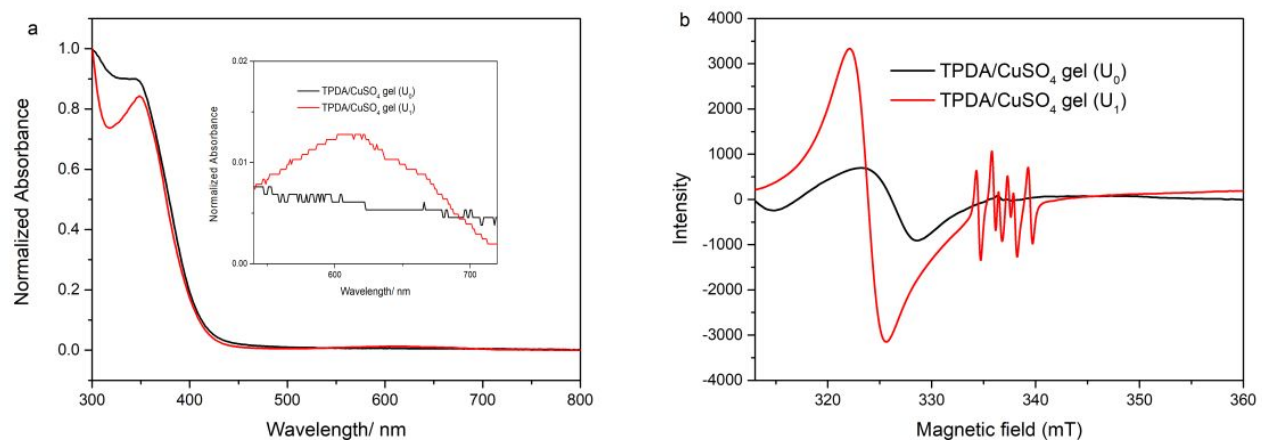

Figure S14 UV-vis and EPR spectra of the gel (TPDA/CuSO 4$)$ by ultrasound in $\mathrm{THF} / \mathrm{H}_{2} \mathrm{O}\left(\mathrm{U}_{0}\right.$ : the gel formed initially by ultrasound, $\mathrm{U}_{1}$ : the gel stood at r.t. for $4 \mathrm{~h}$ ) 


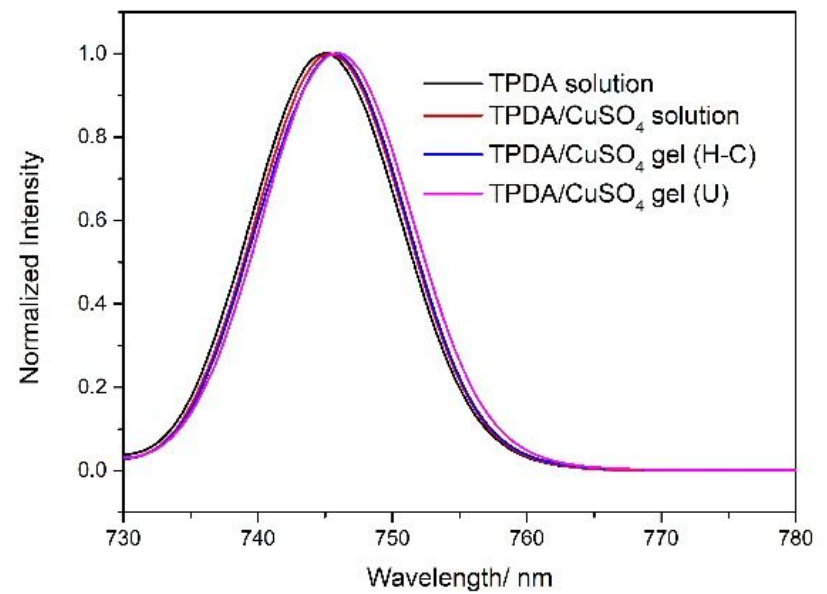

Figure S15 Fluorescent spectra of the solutions and the gels 

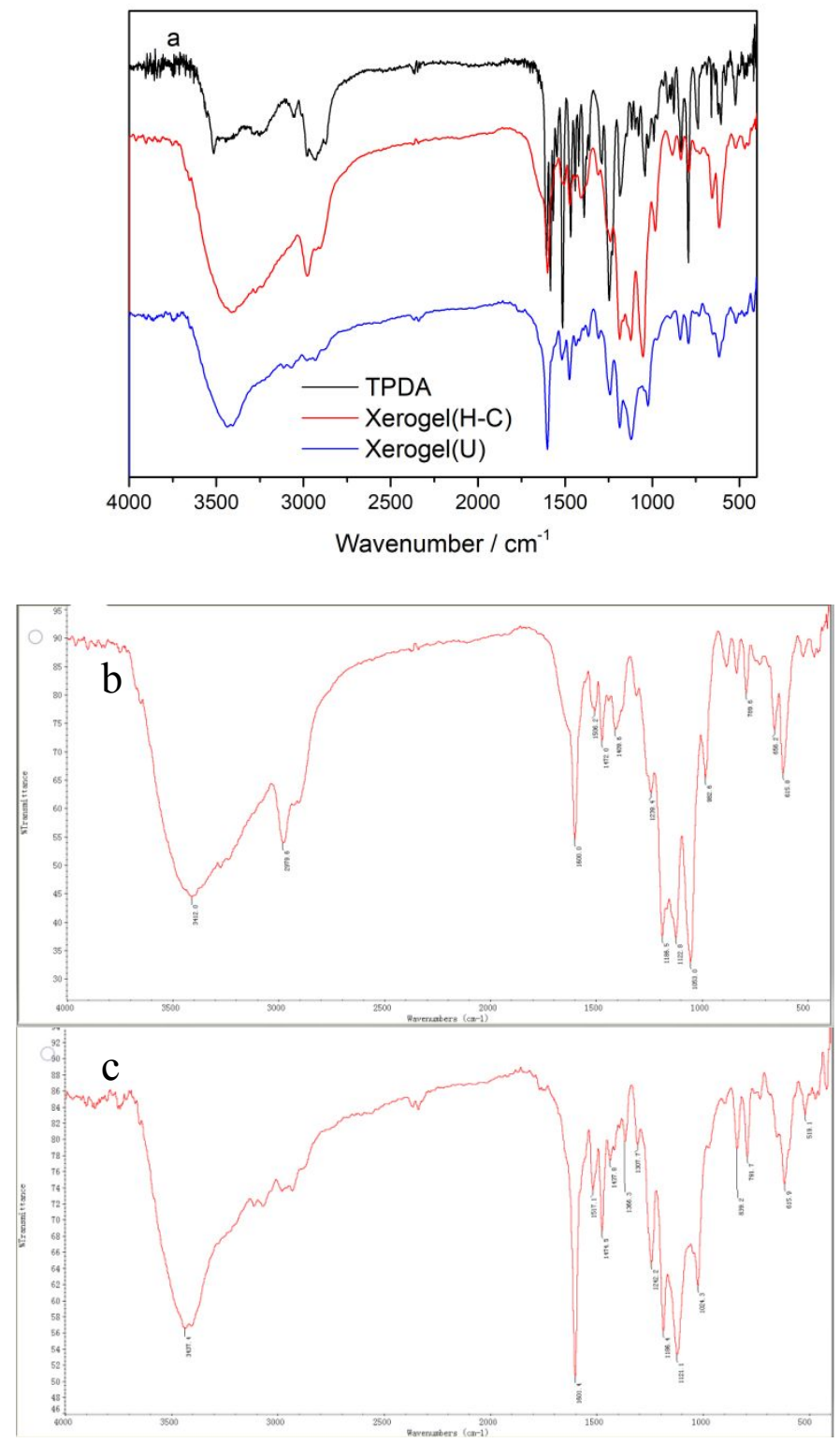

Figure S16 FTIR spectrum of (a) TPDA, xerogel(H-C), xerogel(U); (b) only xerogel(H-C) for clear presentation; (c) only xerogel(U) for clear presentation; 


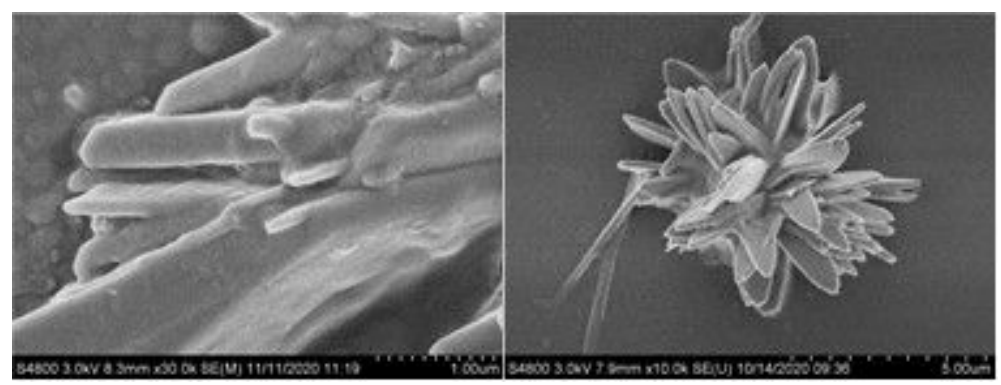

Figure S17 SEM images of the gels(H-C)

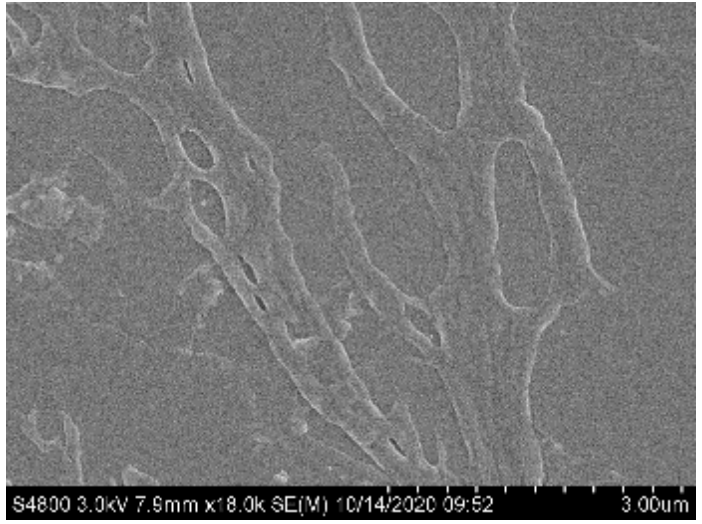

Figure S18 SEM images of the gels(U)

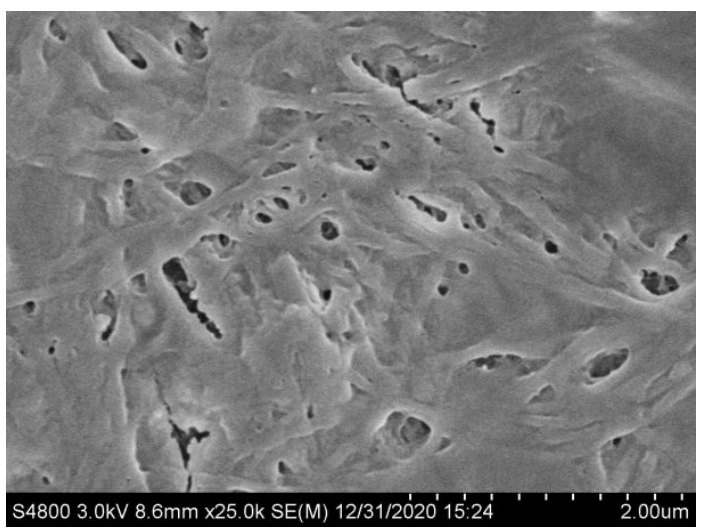

Figure S19 SEM images of the gels formed in $\mathrm{THF} / \mathrm{H}_{2} \mathrm{O}$ by ultrasound 


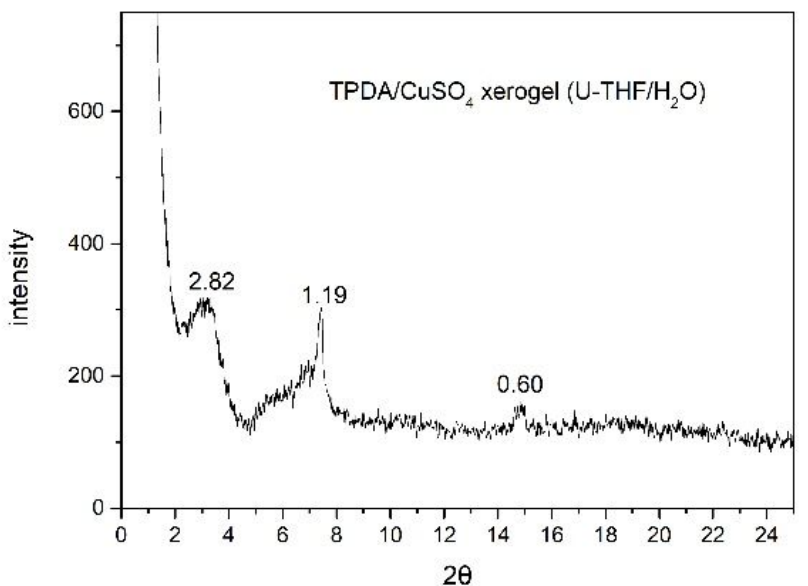

Figure S20 XRD spectra of TPDA/CuSO 4 xerogels $\left(\mathrm{U}-\mathrm{THF} / \mathrm{H}_{2} \mathrm{O}\right)$
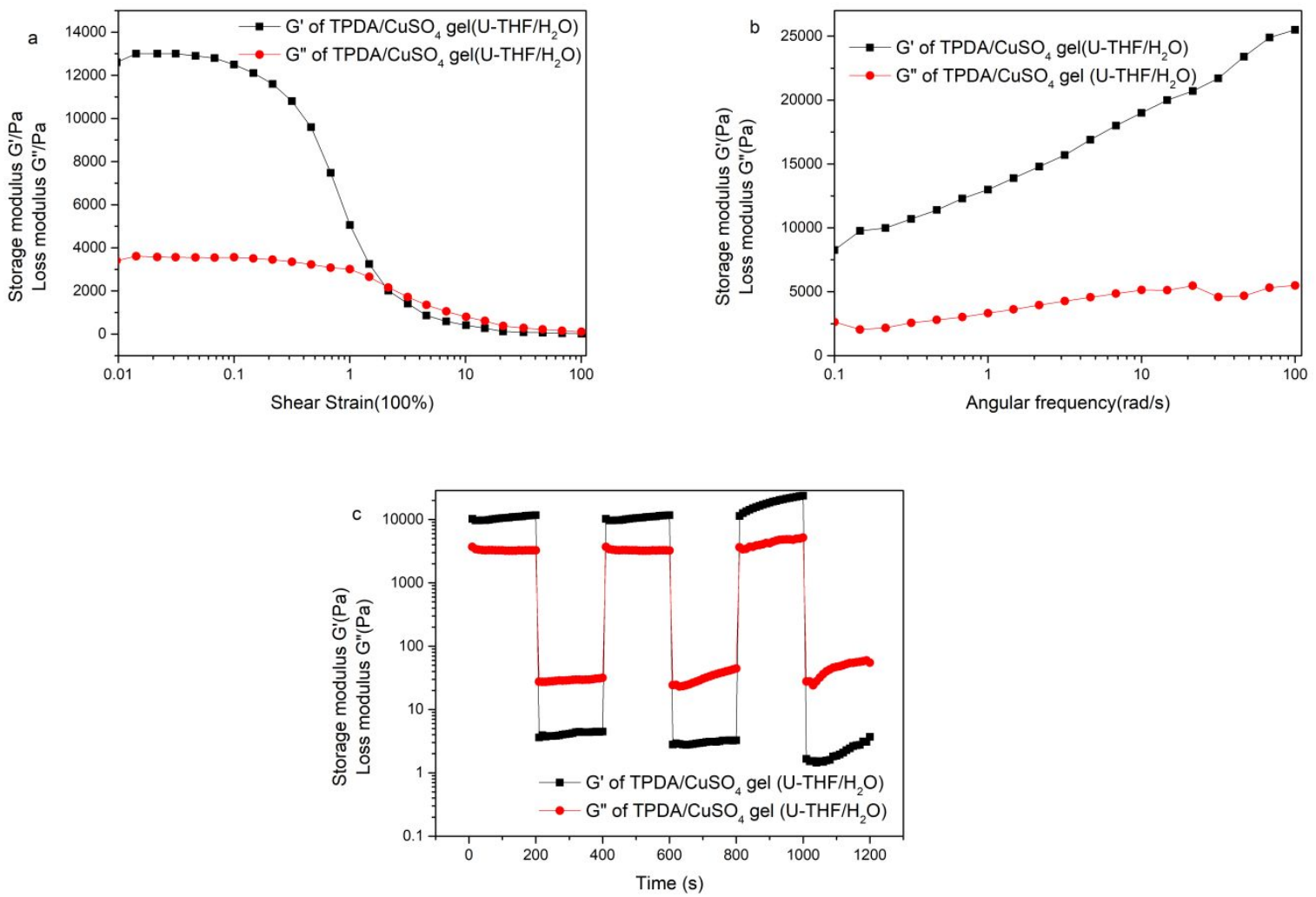

Figure S21 (a) Frequency dependency of $\mathrm{G}^{\prime}$ and $\mathrm{G}^{\prime \prime}$ for TPDA$/ \mathrm{CuSO}_{4}$ gel (TPDA: $25 \mathrm{mg} / \mathrm{mL}$, strain: 0.1\%);

(b) dynamic strain sweep measurement for TPDA/CuSO 4 gel (TPDA: $25 \mathrm{mg} / \mathrm{mL}$, angular frequency at 10 $\mathrm{rad} / \mathrm{s}$ ); (c) recovery test for corresponding $\mathbf{T P D A} / \mathrm{CuSO}_{4}$ gel 


\section{Fabrication of modified electrodes}

Before modification, the GCE was polished carefully with 0.3 and $0.05 \mu \mathrm{m} \alpha$-alumina powder sequentially, and rinsed thoroughly with doubly distilled water and ethanol, respectively. Subsequently, $6 \mu \mathrm{L}$ of solution or gel (the mole ratio of TPDA $\left.\left(25 \mathrm{mgL}^{-1}\right) / \mathrm{Cu}^{2+}=1: 2\right)$ was coated onto the surface of the GCE and dried naturally. The modified GCE was directly used for the determination of $\mathrm{CV}$.

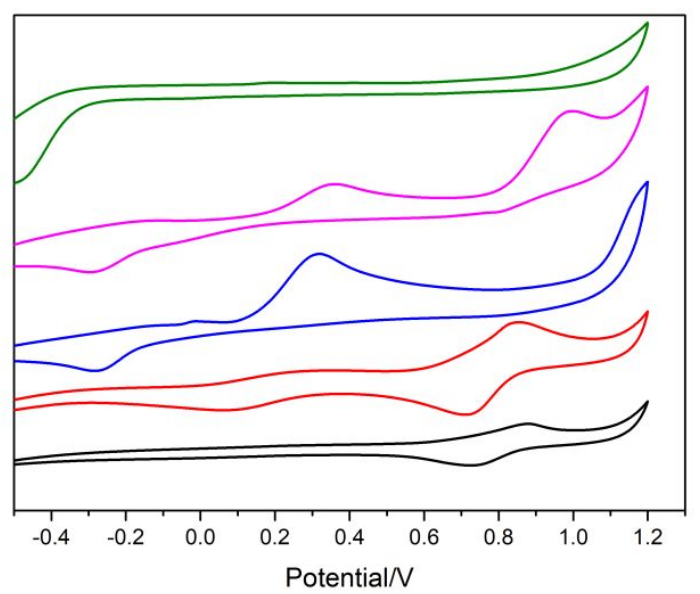

Figure S22 CVs of bare GCE (green), TPDA / GCE (black), TPDA/CuSO 4 / GCE (red), TPDA/CuSO gel $^{2}$ (H-C)/ GCE (purple) and TPDA/CuSO 4 gel (U) / GCE (rose-purple) recorded in $0.1 \mathrm{M} \mathrm{KCl}$ solutions containing $0.05 \mathrm{M} \mathrm{Na}_{2} \mathrm{HPO}_{4}+\mathrm{NaH}_{2} \mathrm{PO}_{4}$ (PBS)
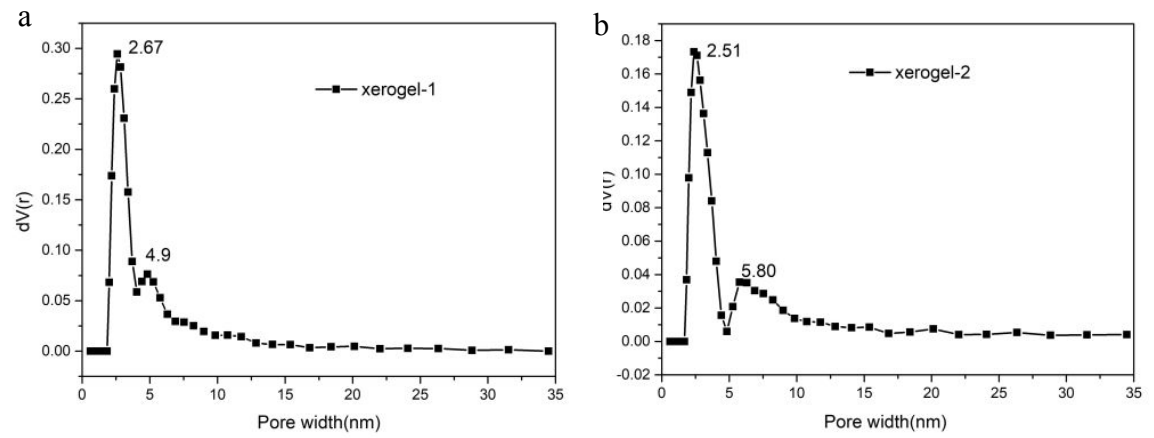
Figure S23 Pore size distribution of xerogel-1 (a) and xerogel-2 (b) (Model: $\mathrm{N}_{2}$ at $77 \mathrm{~K}$ on carbon)

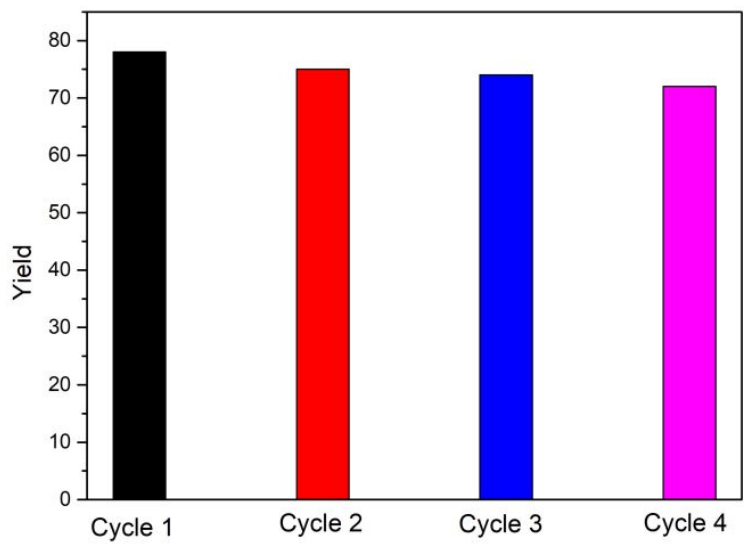

Figure S24 Reusability of the xerogel-1 catalyst in CuAAC reaction between benzyl azide and

phenylacetylene

\section{General procedures of $\mathrm{CuAAC}$ reaction}

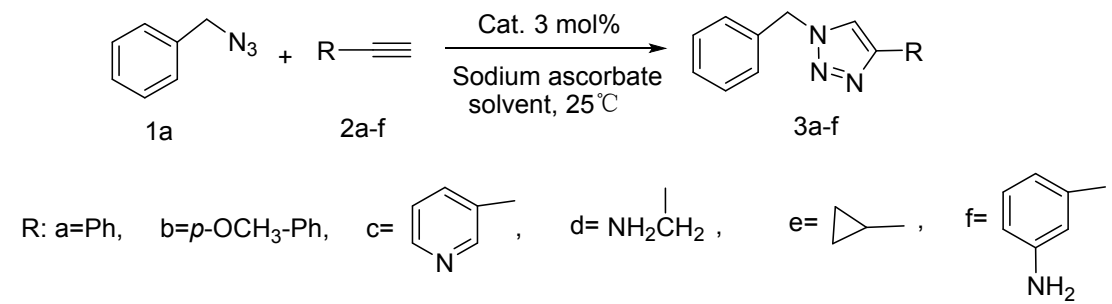

1-Benzyl-4-phenyl-1H-1,2,3-triazole (3a, CAS No: 108717-96-0) ${ }^{[4]}$ : The crude product was purified by column chromatography on silica gel using dichloromethane: ethyl acetate (10: 1). White solid, m.p. $125-126^{\circ} \mathrm{C}$; IR(KBr): $3136,1663,1464,1228,773,733,689 \mathrm{~cm}^{-1}$; ${ }^{1} \mathrm{H}$ NMR $(500$ $\left.\mathrm{MHz}, \mathrm{CDCl}_{3}\right) \delta(\mathrm{ppm}): 7.81(\mathrm{~d}, J=1.5 \mathrm{~Hz}, 2 \mathrm{H}, \mathrm{Ph}), 7.66(\mathrm{~s}, 1 \mathrm{H}, \mathrm{CH}=\mathrm{C}), 7.36-7.42(\mathrm{~m}, 5 \mathrm{H}, \mathrm{Ph})$, 7.30-7.33 (m, 2H, Ph), $\left.5.58\left(\mathrm{~s}, 2 \mathrm{H},-\mathrm{CH}_{2}\right) ;{ }^{13} \mathrm{C} \mathrm{NMR} \mathrm{(125} \mathrm{MHz,} \mathrm{CDCl}_{3}\right) \delta(\mathrm{ppm}): 148.2,134.7$, $130.6,129.2,128.8,128.7,128.2,128.1,125.7,119.6,54.2$. 


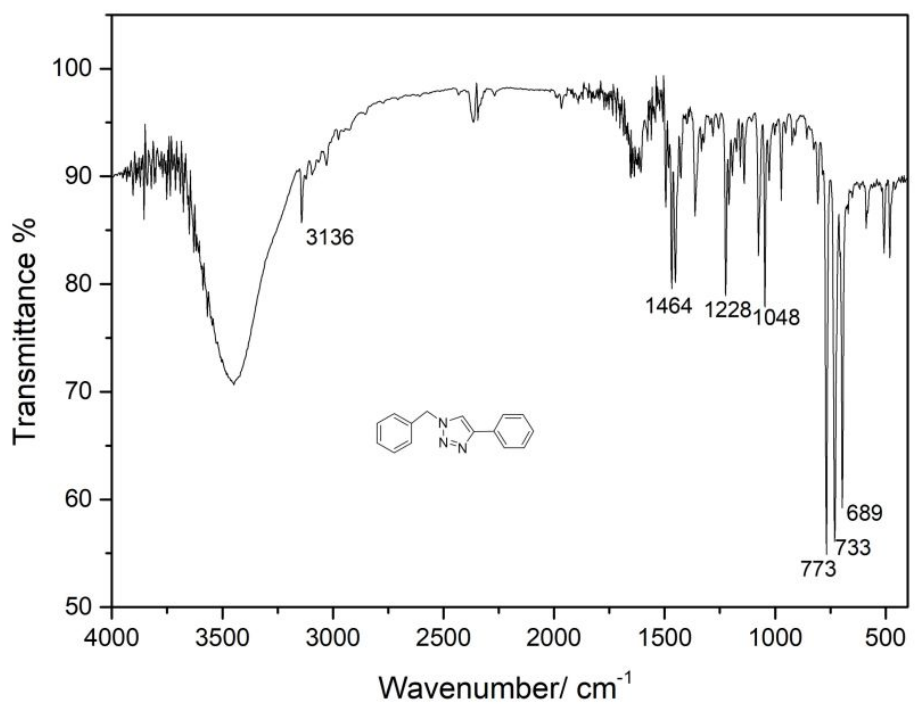

IR spectra of compound $\mathbf{3 a}$

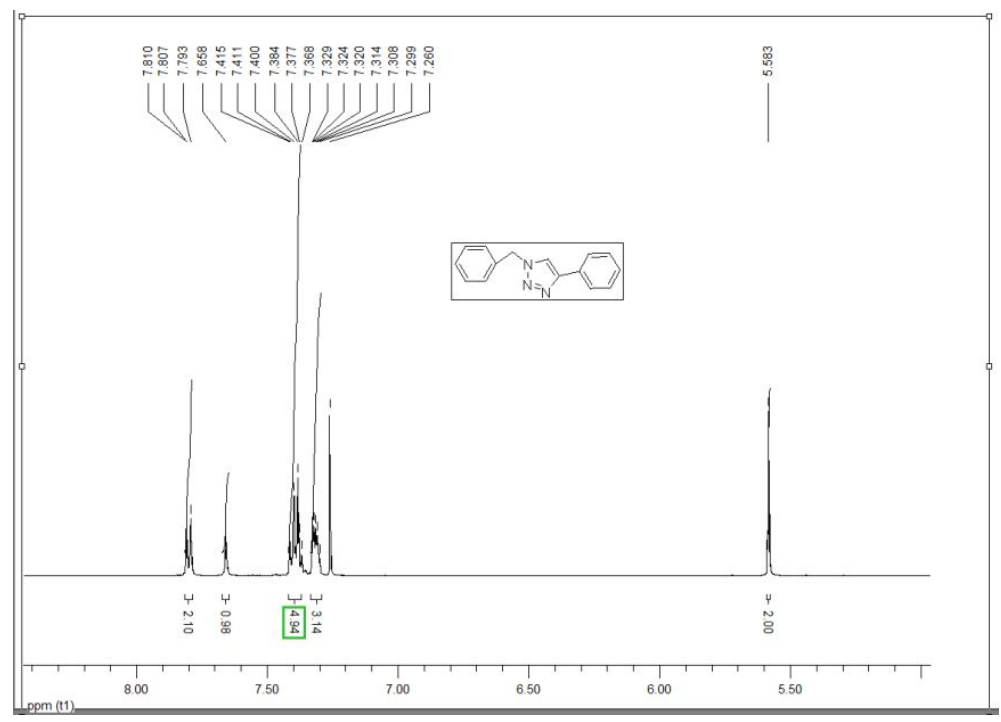

${ }^{1} \mathrm{H}$ NMR spectra of compound $\mathbf{3 a}$ 


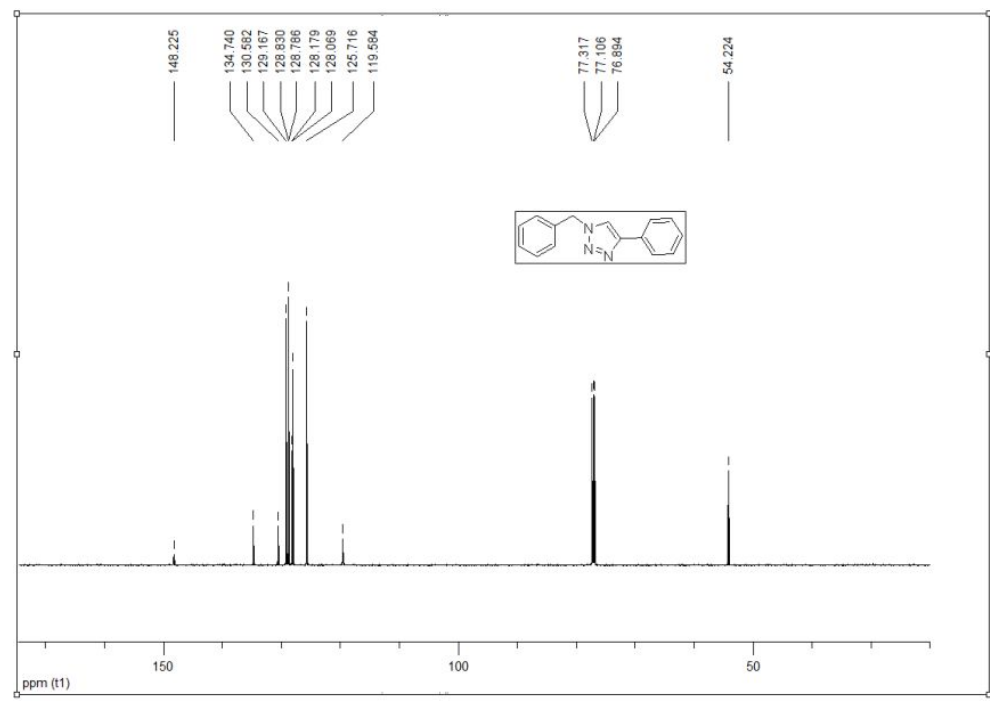

${ }^{13} \mathrm{C}$ NMR spectra of compound $\mathbf{3 a}$

1-Benzyl-4-(4-methoxyphenyl)-1H-1,2,3-triazole (3b, CAS No: 116557-81-43) ${ }^{[4]}$ : The crude product was purified by column chromatography on silica gel using dichloromethane: ethyl acetate (10: 1). White solid, m.p. $146-147^{\circ} \mathrm{C}$; IR(KBr): 3140, 2926, 2839, 1621, 1495, 1250, 838, 729 $\mathrm{cm}^{-1} ;{ }^{1} \mathrm{H}$ NMR $\left(500 \mathrm{MHz}, \mathrm{CDCl}_{3}\right) \delta(\mathrm{ppm}): 7.73(\mathrm{~d}, J=8.5 \mathrm{~Hz}, 2 \mathrm{H}, \mathrm{Ph}), 7.57(\mathrm{~s}, 1 \mathrm{H}, \mathrm{CH}=\mathrm{C}), 7.36-$ $7.39(\mathrm{~m}, 3 \mathrm{H}, \mathrm{Ph}), 7.30-7.31(\mathrm{~m}, 2 \mathrm{H}, \mathrm{Ph}), 6.92(\mathrm{~d}, J=8.5 \mathrm{~Hz}, 2 \mathrm{H}, \mathrm{Ph}), 5.56\left(\mathrm{~s}, 2 \mathrm{H},-\mathrm{CH}_{2}\right) ;{ }^{13} \mathrm{C} \mathrm{NMR}$ $\left(125 \mathrm{MHz}, \mathrm{CDCl}_{3}\right) \delta(\mathrm{ppm}): 159.6,148.1,134.8,129.1,128.7,128.0,127.0,123.3,118.8,114.2$ $55.3,54.2$. 


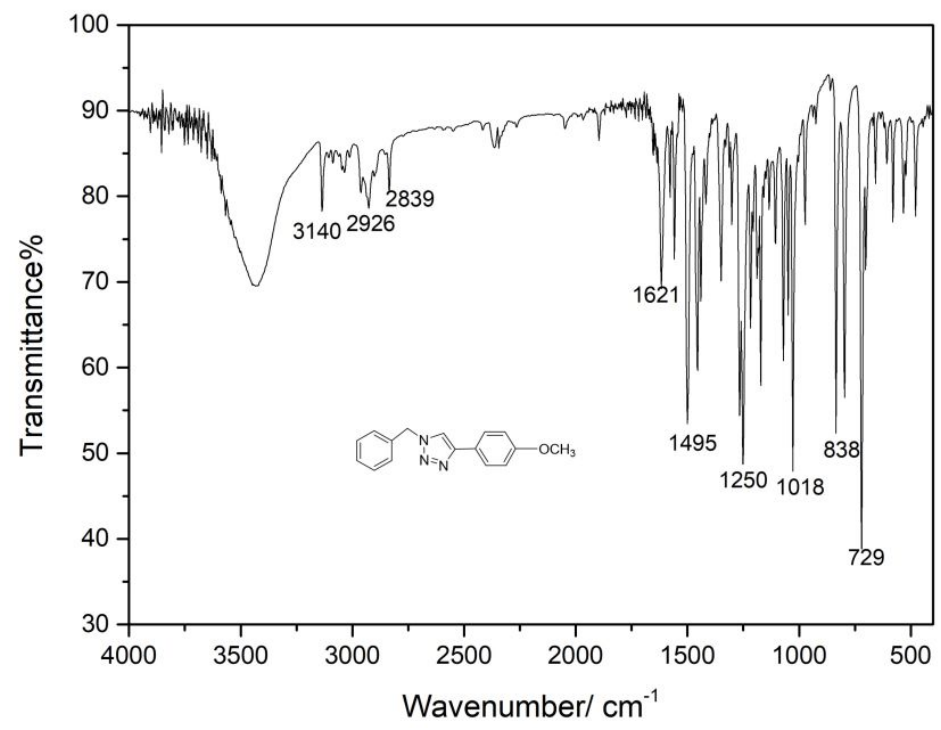

IR spectra of compound $\mathbf{3 b}$

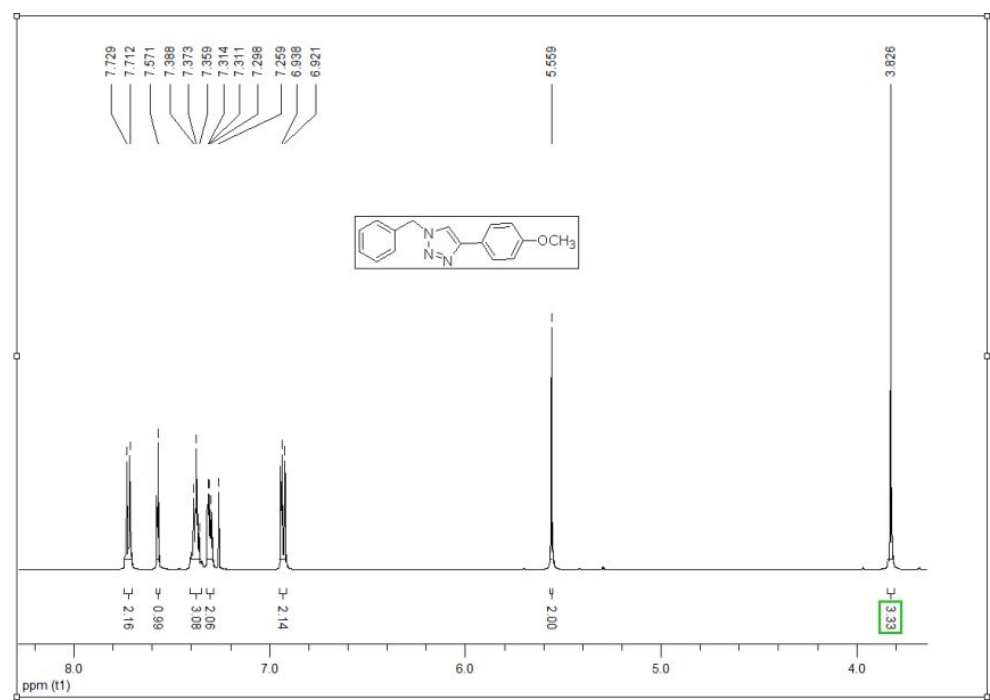

${ }^{1} \mathrm{H}$ NMR spectra of compound $\mathbf{3 b}$ 


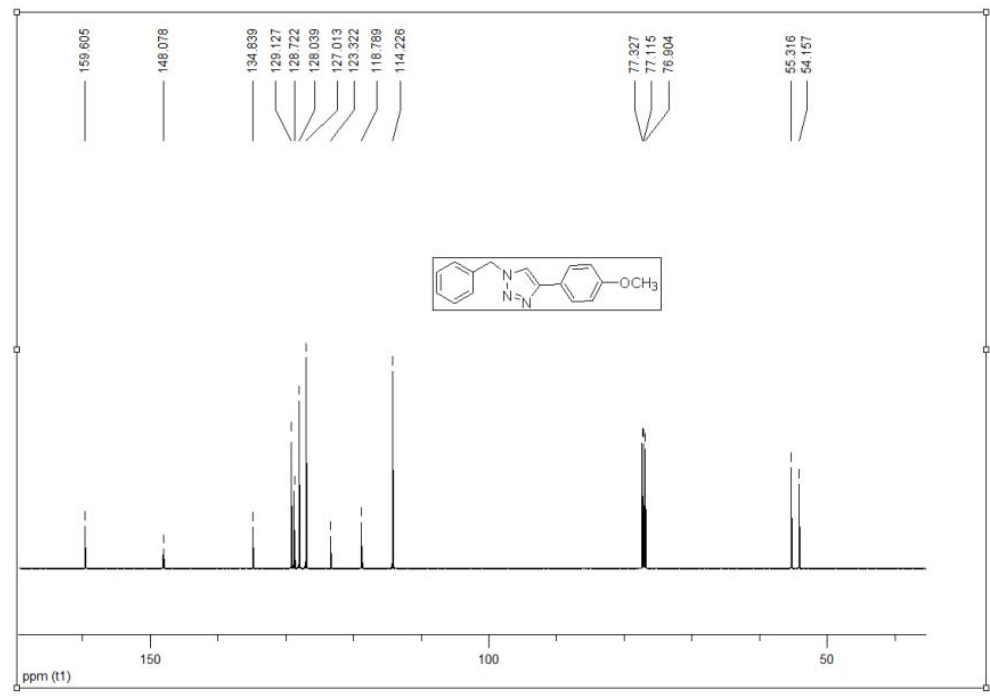

${ }^{13} \mathrm{C}$ NMR spectra of compound $\mathbf{3 b}$

3-(1-Benzyl-1H-1,2,3-triazol-4-yl)pyridine (3c, CAS No: 1034194-35-8) ${ }^{[5]}$ : The crude product was purified by column chromatography on silica gel using dichloromethane: ethyl acetate (3: 1). White solid, m.p. $100-101{ }^{\circ} \mathrm{C}$; IR(KBr): $3110,1576,1451,1219,807,728,698 \mathrm{~cm}^{-1} ;{ }^{1} \mathrm{H}$ NMR $(500$ $\left.\mathrm{MHz}, \mathrm{CDCl}_{3}\right) \delta(\mathrm{ppm}): 8.95(\mathrm{~d}, J=1.5 \mathrm{~Hz}, 1 \mathrm{H}, \mathrm{Py}), 8.55-8.56\left(\mathrm{dd}, J_{1}=4.5 \mathrm{~Hz}, J_{2}=1.5 \mathrm{~Hz}, 1 \mathrm{H}\right.$, Py), 7.74(s, 1H, CH=C), 7.38-7.42 (m, 3H, Ph), 7.32-7.35 (m, 2H, Ph), $5.60\left(\mathrm{~s}, 2 \mathrm{H},-\mathrm{CH}_{2}\right) .{ }^{13} \mathrm{C}$ NMR (125 MHz, $\left.\mathrm{CDCl}_{3}\right) \delta(\mathrm{ppm}): 149.3,147.0,145.2,134.4,132.9,129.2,128.9,128.1,126.7$, 123.7, 119.9, 54.4 . 


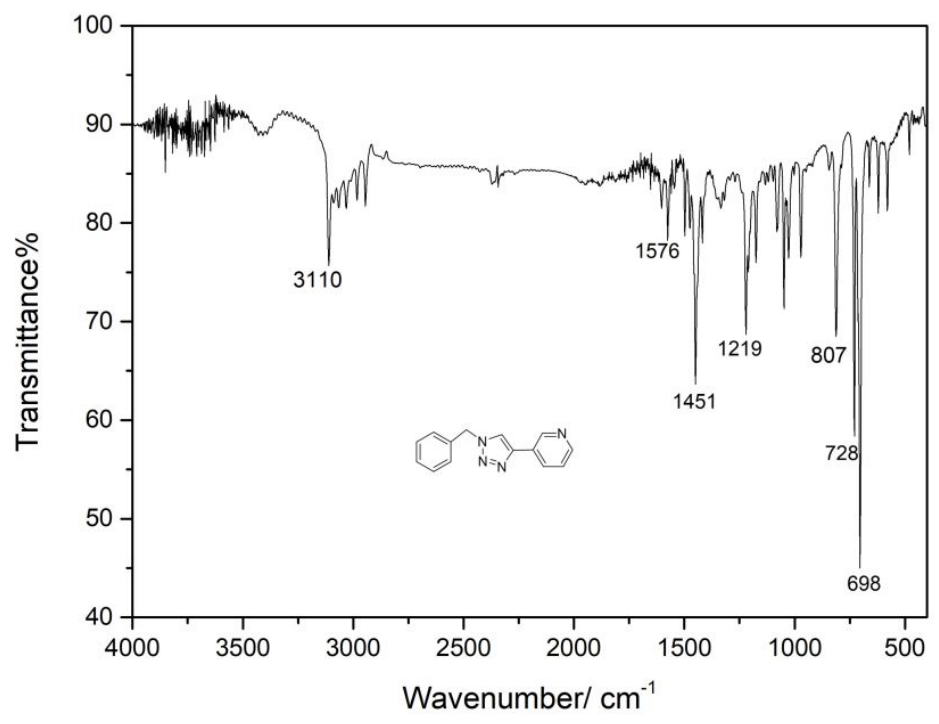

IR spectra of compound 3c

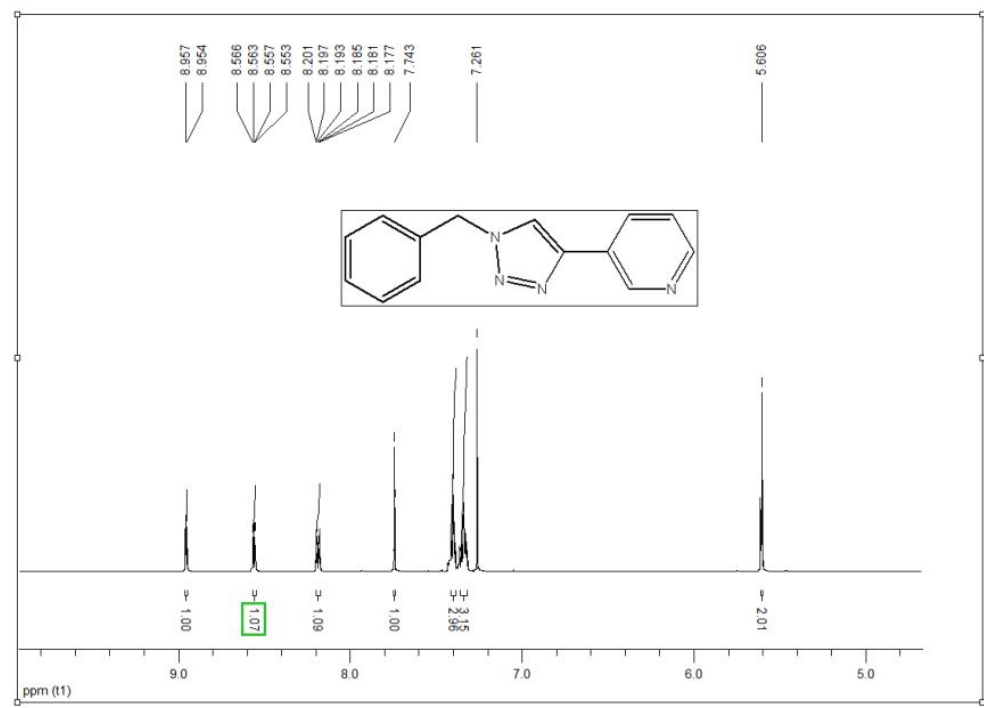

${ }^{1} \mathrm{H}$ NMR spectra of compound $\mathbf{3 c}$ 


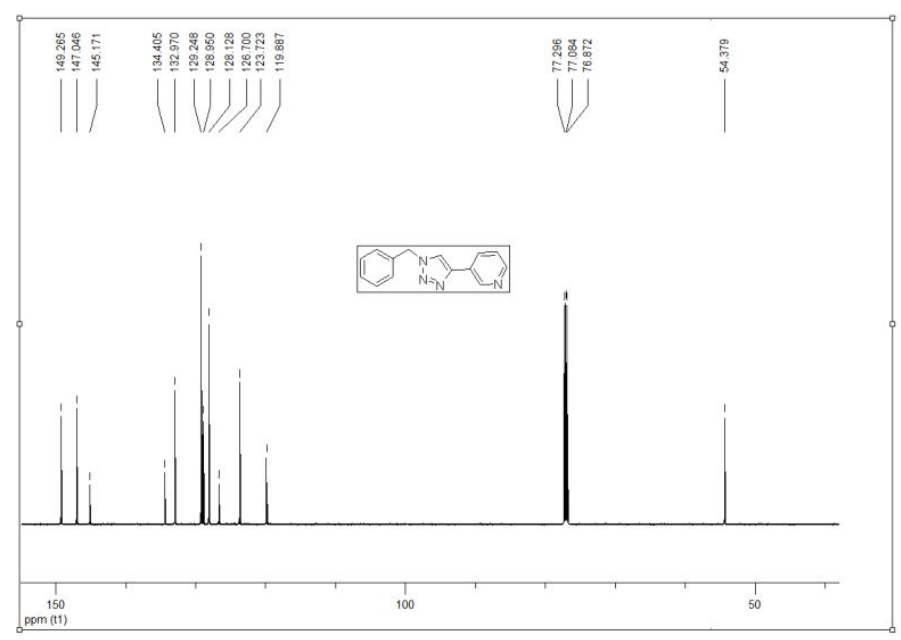

${ }^{13} \mathrm{C}$ NMR spectra of compound $\mathbf{3 c}$

1-Benzyl-1H-1,2,3-triazol-4-yl-methanamine(3d, CAS No: $760952-78-1)^{[6]}$ The crude product was purified by column chromatography on silica gel using methanol: triethylamine (10: 1). Pale yellow solid, m.p. $107-109^{\circ} \mathrm{C}$; IR(KBr): 3457, 3420, 3102, 3039, 1659, 1570, 1451, 1051, 713 $\mathrm{cm}^{-1} ;{ }^{1} \mathrm{H}$ NMR $\left(500 \mathrm{MHz}, \mathrm{DMSO}-d_{6}\right) \delta(\mathrm{ppm}): 7.93(\mathrm{~s}, 1 \mathrm{H}, \mathrm{CH}=\mathrm{C}), 7.30-7.39(\mathrm{~m}, 5 \mathrm{H}, \mathrm{Ph}), 5.56$ $\left(\mathrm{s}, 2 \mathrm{H},-\mathrm{CH}_{2}\right), 3.76\left(\mathrm{~s}, 2 \mathrm{H},-\mathrm{CH}_{2}\right) ;{ }^{13} \mathrm{C} \mathrm{NMR}\left(125 \mathrm{MHz}, \mathrm{CDCl}_{3}\right) \delta(\mathrm{ppm}): 155.3,146.3,133.9$, 129.1, 128.3, 122.8, 56.1, 54.2.

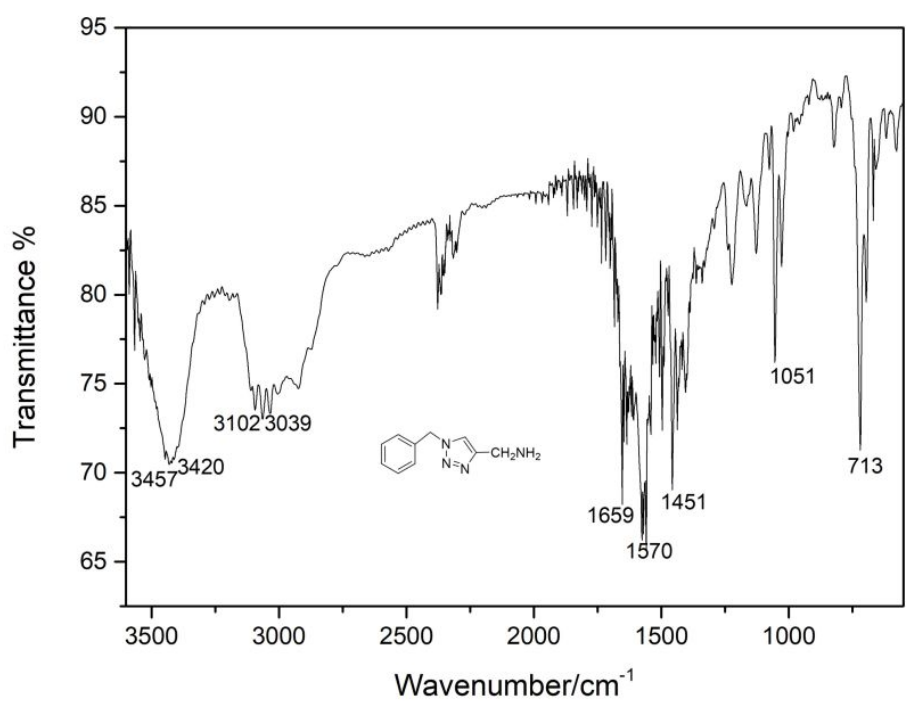

IR spectra of compound 3d 


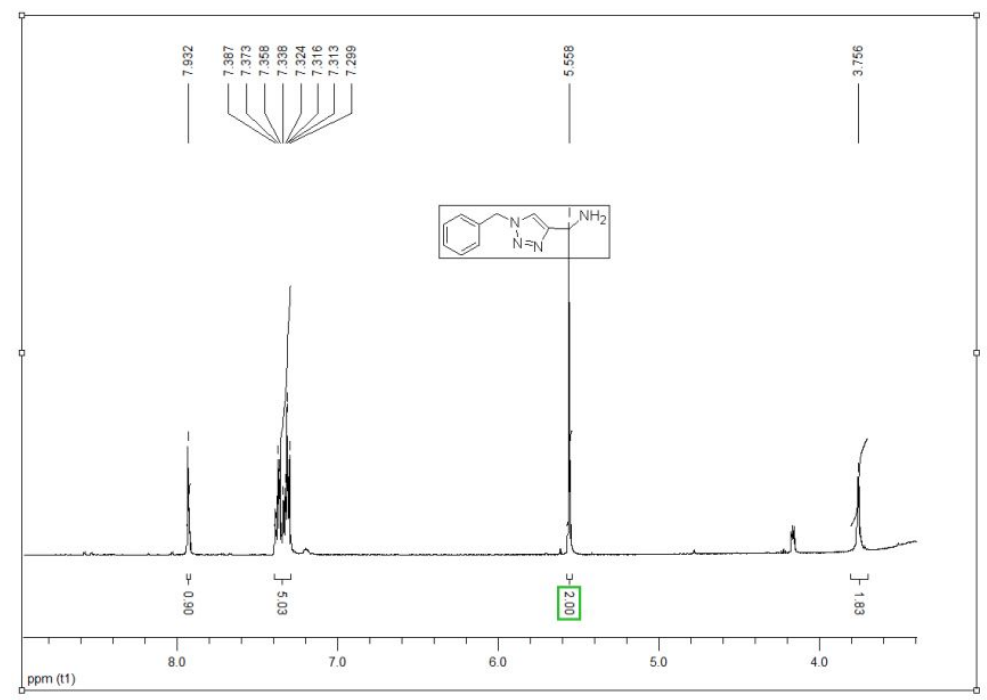

${ }^{1} \mathrm{H}$ NMR spectra of compound $\mathbf{3 d}$

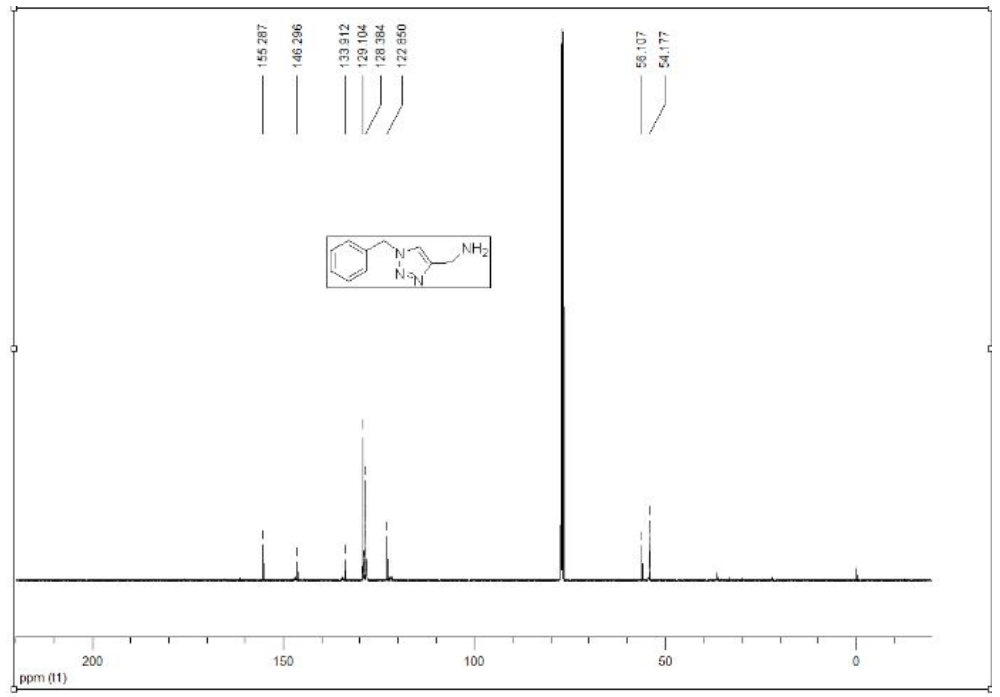

${ }^{13} \mathrm{C}$ NMR spectra of compound $\mathbf{3 d}$

1-Benzyl-4-cyclopropyl-1H-1,2,3-triazole (3e, CAS No: 1151920-05-6) ${ }^{[5]}$ : The crude product was purified by column chromatography on silica gel using petroleum ether: ethyl acetate (2: 1). White solid, m.p. $73-75^{\circ} \mathrm{C}$; IR(KBr): 3080, 1565, 1459, 1215, 1022, $721 \mathrm{~cm}^{-1}$; ${ }^{1} \mathrm{H}$ NMR (500 MHz, $\left.\mathrm{CDCl}_{3}\right) \delta(\mathrm{ppm}):$ 7.32-7.38 (m, 3H, Ph), 7.24-7.26 (m, 2H, Ph), $7.13(\mathrm{~s}, 1 \mathrm{H}, \mathrm{CH}=\mathrm{C}), 5.46(\mathrm{~s}, 2 \mathrm{H}$, $\left.-\mathrm{CH}_{2}\right), 1.89-1.93(\mathrm{~m}, 1 \mathrm{H},-\mathrm{CH}), 1.26-1.34\left(\mathrm{~m}, 4 \mathrm{H},-2 \mathrm{CH}_{2}\right) ;{ }^{13} \mathrm{C} \mathrm{NMR}\left(125 \mathrm{MHz}, \mathrm{CDCl}_{3}\right) \delta(\mathrm{ppm})$ : 
150.6, 134.9, 129.0, 128.6, 127.9, 119.6, 54.9, 7.7, 6.7.

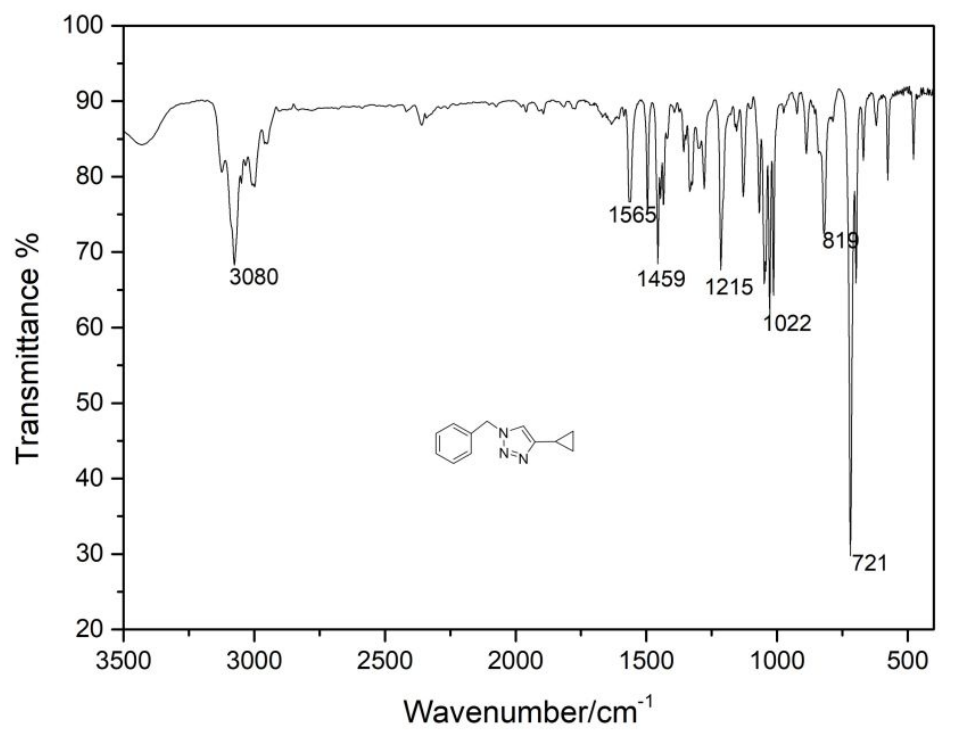

IR spectra of compound $\mathbf{3 e}$

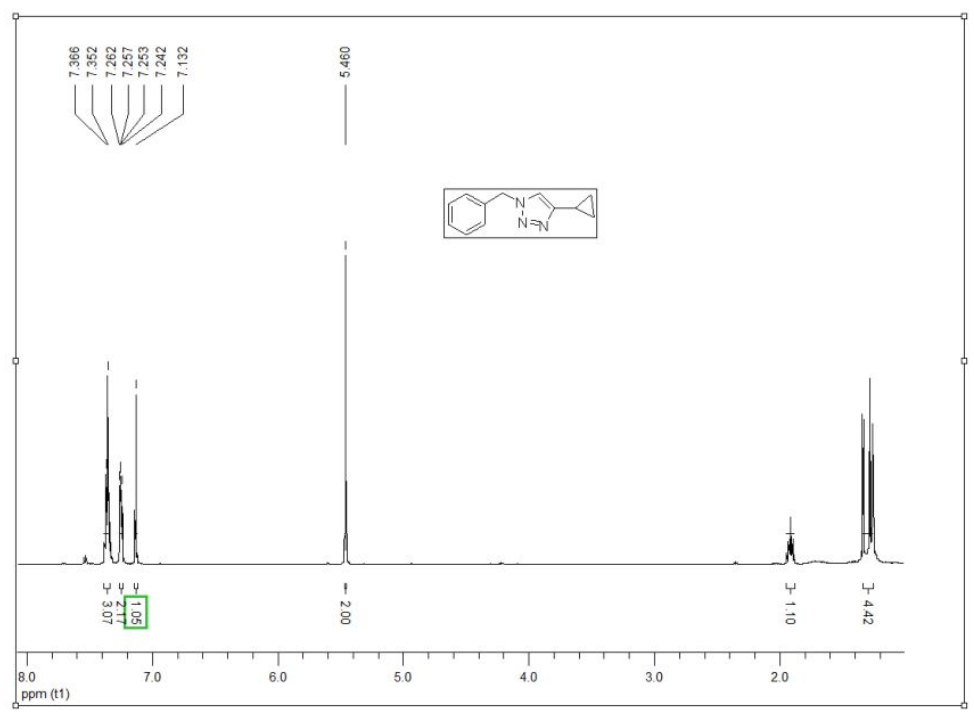

${ }^{1} \mathrm{H}$ NMR spectra of compound $\mathbf{3 e}$ 


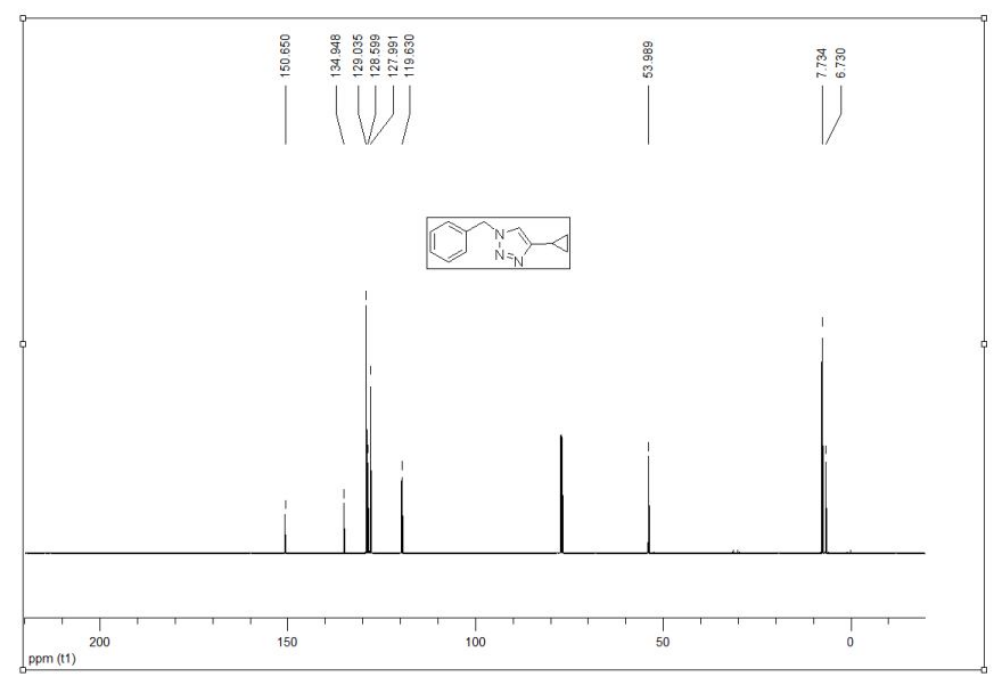

${ }^{13} \mathrm{C}$ NMR spectra of compound $\mathbf{3 e}$

3-(1-phenyl-1H-1,2,3-triazol-4-yl)aniline(3f, CAS No: 1151920-00-1) ${ }^{[7]}$ : The crude product was purified by column chromatography on silica gel using petroleum ether: ethyl acetate (1: 1). Pale yellow solid, m.p. $123-125^{\circ} \mathrm{C}$; IR(KBr): 3451, 3372, 1614, 1481, 1052, 785, $729 \mathrm{~cm}^{-1}$; ${ }^{1} \mathrm{H}$ NMR $\left(500 \mathrm{MHz}, \mathrm{DMSO}-d_{6}\right) \delta(\mathrm{ppm}): 7.61(\mathrm{~s}, 1 \mathrm{H}, \mathrm{CH}=\mathrm{C}), 7.39-7.41(\mathrm{~m}, 3 \mathrm{H}, \mathrm{Ph}), 7.29-7.31(\mathrm{~m}, 2 \mathrm{H}$, $\mathrm{Ph}), 7.24(\mathrm{t}, J=2.0 \mathrm{~Hz}, 1 \mathrm{H}), 7.17(\mathrm{t}, J=7.5 \mathrm{~Hz}, 1 \mathrm{H}), 7.09(\mathrm{~m}, 1 \mathrm{H}, \mathrm{Py}), 5.57(\mathrm{~s}, 2 \mathrm{H}), 3.74(\mathrm{bs}, 2 \mathrm{H}$, $\left.-\mathrm{NH}_{2}\right) .{ }^{13} \mathrm{C} \mathrm{NMR}\left(125 \mathrm{MHz}, \mathrm{CDCl}_{3}\right) \delta(\mathrm{ppm}): 148.3,134.7,131.5,129.7,129.2,128.7,128.1$, 119.5, 116.2, 115.1, 112.4, 54.2. 


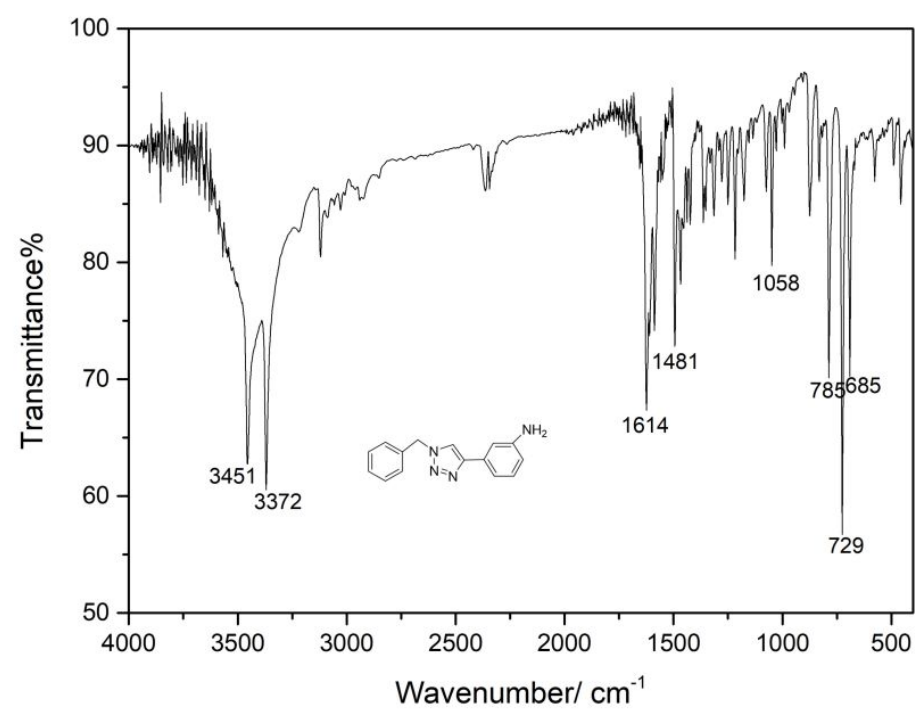

IR spectra of compound $\mathbf{3 f}$

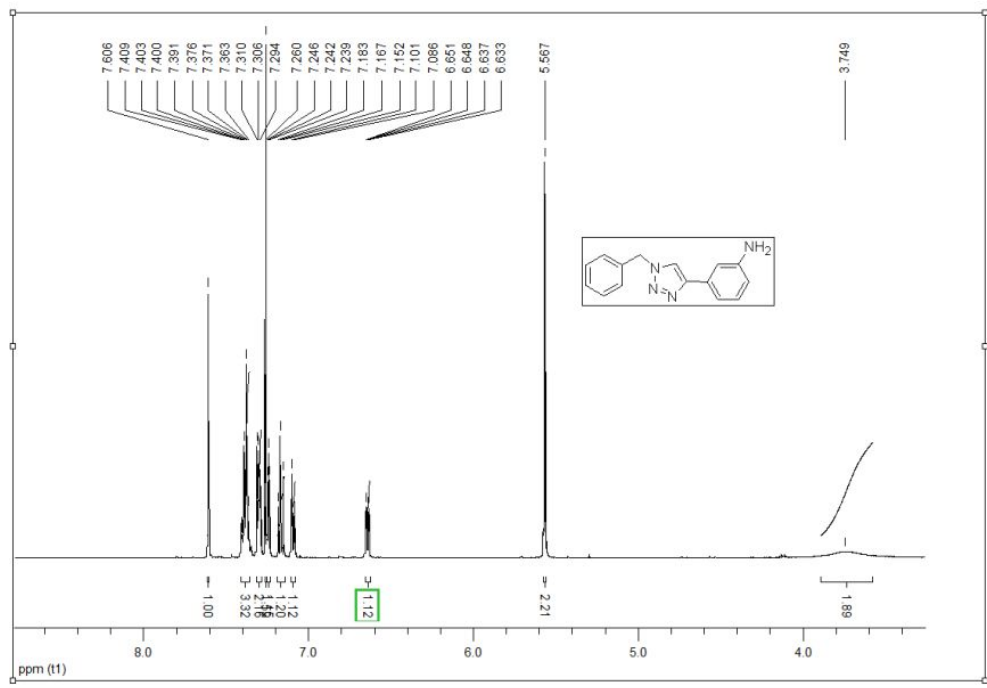

${ }^{1} \mathrm{H}$ NMR spectra of compound $\mathbf{3 f}$ 


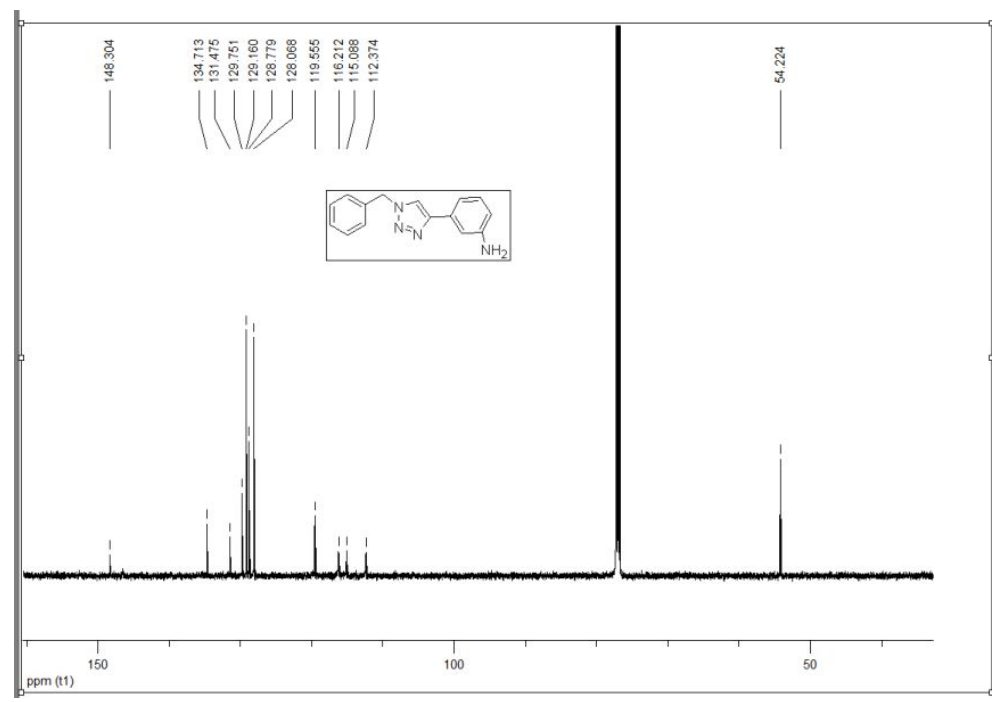

${ }^{13} \mathrm{C}$ NMR spectra of compound $\mathbf{3 f}$

(1) Wang, S.; Chu, W.; Wang, Y.; Liu, S.; Zhang, J.; Li, S.; Wei, H.; Zhou, G.; Qin, X. Synthesis, characterization and cytotoxicity of $\mathrm{Pt}(\mathrm{II}), \mathrm{Pd}(\mathrm{II}), \mathrm{Cu}(\mathrm{II})$ and $\mathrm{Zn}(\mathrm{II})$ complexes with 4'-substituted terpyridine. Appl. Organometal. Chem. 2013, 27, 373-379.

(2) Che, X,; Sheng, C.; Wang, W.; Cao, Y.; Xu, Y.; Ji, H.; Dong, G.; Miao, Z.; Yao, J.; Zhang, W. New azoles with potent antifungal activity: Design, synthesis and molecular docking. Eur. J. Med. Chem. 2009, 44, 42184226.

(3) Hirel, C.; Vostrikova, K. E.; Pécaut, J.; Ovcharenko, V. I.; Rey, P. Nitronyl and imino nitroxides: improvement of ullman's procedure and report on a new efficient synthetic route. Chem. Eur. J. 2001, 7, 20072014.

(4) Lim, M.; Lee, H.; Kang, M.; Yoo, W.; Rhee, Hakjune. Azide-alkyne cycloaddition reactions in water via recyclable heterogeneous $\mathrm{Cu}$ catalysts: reverse phase silica gel and thermoresponsive hydrogels. $R S C$ adv. 2018 , $8,6152-6159$.

(5) Sun, N.; Yu, Z.; Yi, H.; Zhu, X.; Jin, L.; Hu, B.; Shen, Z.; Hu, X. Synthesis of a heterogeneous Cu(OAc) $2_{2}^{-}$ 
anchored SBA-15 catalyst and its application in CuAAC reaction. New J. Chem., 2018, 42, 1612-1616.

(6) Taskin, O. S.; Dadashi-Silab, S.; Kiskan, B.; Weber, J.; Yagci, Y. Highly efficient and reusable microporous schiff base network polymer as a heterogeneous catalyst for CuAAC click reaction. Macromol. Chem. Phys. 2015, 216, 1746-1753.

(7) Jiang, Y.; Li, X.; Li, X.; Sun, Y.; Zhao, Y.; Jia, S.; Guo, N.; Xu, G.; Zhang, W. Copper(II) acetylacetonate: an efficient catalyst for Huisgen-click reaction for synthesis of 1,2,3-triazoles in water. Chin. J. Chem. 2017, 35, $1239-1245.7$ 